Small-scale instabilities in dynamical systems with sliding

Sieber, J and Kowalczyk, P

2010

MIMS EPrint: 2010.2

Manchester Institute for Mathematical Sciences

School of Mathematics

The University of Manchester

\footnotetext{
Reports available from: http://eprints.maths.manchester.ac.uk/

And by contacting: The MIMS Secretary

School of Mathematics

The University of Manchester

Manchester, M13 9PL, UK
} 


\title{
Small-scale instabilities in dynamical systems with sliding
}

\author{
J. Sieber ${ }^{\text {a,* }}$, P. Kowalczyk ${ }^{\text {b }}$ \\ a Department of Mathematics, University of Portsmouth, Portsmouth, PO1 3HF, UK \\ ${ }^{\mathrm{b}}$ School of Mathematics, University of Manchester, Manchester, M13 9PL, UK
}

\section{A R T I C L E I N F O}

\section{Article history:}

Received 12 November 2008

Received in revised form

21 July 2009

Accepted 4 October 2009

Available online 22 October 2009

Communicated by G. Stepan

\section{Keywords:}

Vector fields with sliding

Singular perturbation

Discontinuity induced bifurcation

\begin{abstract}
A B S T R A C T
We demonstrate with a minimal example that in Filippov systems (dynamical systems governed by discontinuous but piecewise smooth vector fields) stable periodic motion with sliding is not robust with respect to stable singular perturbations. We consider a simple dynamical system that we assume to be a quasi-static approximation of a higher-dimensional system containing a fast stable subsystem. We tune a system parameter such that a stable periodic orbit of the simple system touches the discontinuity surface: this is the so-called grazing-sliding bifurcation. The periodic orbit remains stable, and its local return map becomes piecewise linear. However, when we take into account the fast dynamics the local return map of the periodic orbit changes qualitatively, giving rise to, for example, period-adding cascades or small-scale chaos.
\end{abstract}

(c) 2009 Elsevier B.V. All rights reserved.

\section{Introduction}

Filippov systems are dynamical systems governed by discontinuous but piecewise smooth ordinary differential equations (ODEs) and they occur, for example, in the modelling of mechanical systems (with dry friction) [1], of electrical systems with switching [2], or of population dynamics (when, depending on the population size, either the individuals switch habitat or diet, or harvesting becomes restricted [3]). The phase space of a Filippov system is partitioned into domains where for each domain a different ODE governs the dynamics. In the simplest case we have two domains such that

$\dot{x}= \begin{cases}f_{-}(x) & \text { if } h(x)<0, \\ f_{+}(x) & \text { if } h(x) \geq 0 .\end{cases}$

The boundary between the domains is called the switching manifold: $\mathscr{H}_{s}=\{h(x)=0\}$.

A special feature of Filippov systems is the so-called sliding mode, which means that a trajectory of the system does not follow any of the ODEs governing the domains but it rather 'slides' along the switching manifold, following a convex combination of the ODEs governing the adjacent domains:

$\dot{x}=f_{s}(x)=\frac{\left[\partial h(x) f_{-}(x)\right] \cdot f_{+}(x)-\left[\partial h(x) f_{+}(x)\right] \cdot f_{-}(x)}{\partial h(x)\left[f_{-}(x)-f_{+}(x)\right]}$,

\footnotetext{
* Corresponding author.

E-mail address: jan.sieber@port.ac.uk (J. Sieber).
}

where ' $\partial$ ' denotes partial differentiation (in the current case with respect to the variable $x$ ). Sliding occurs in all points $x_{0}$ of the switching manifold $\mathscr{H}_{s}$ where both vector fields point toward $\mathscr{H}_{s}$, that is where $\partial h(x) f_{+}(x)<0$ and $\partial h(x) f_{-}(x)>0$. Fig. 1 illustrates the two typically observed cases of interaction between flows and switching manifold.

An important question for modelling is how sliding in a Filippov system is affected by perturbations. If we add a small perturbation to $f_{-}$or $f_{+}$or to the switching decision $h$ (the derivative of the perturbation is also assumed to be small) then any exponentially stable periodic orbit or equilibrium of a Filippov system persists [4] and remains stable. This also applies to pseudo-equilibria (equilibria of the sliding flow $f_{s}$, sitting exactly on the switching manifold) and to periodic orbits that have sliding segments. This persistence mirrors the results of classical bifurcation and invariant manifold theory for smooth dynamical systems [5].

Another typical perturbation arising in the modelling process is a stable singular perturbation. In a simple model one has replaced rapidly converging parts of the dynamics with their equilibrium, making the assumption that this equilibrium follows the slow dynamics quasi-statically. In a more complex model of the same system (or in reality) the equilibrium of the fast dynamics is not attained perfectly, which constitutes a small perturbation. Practical examples of this type of perturbation are small capacitances and inductances in electrical circuits, imperfect rigidity in mechanical systems, or fast chemical reactions (or other processes) in biological systems. Again, for smooth dynamical systems classical theory [5] proves that all hyperbolic equilibria, periodic orbits and, more generally, normally hyperbolic invariant manifolds persist. That is, for example, an exponentially stable 


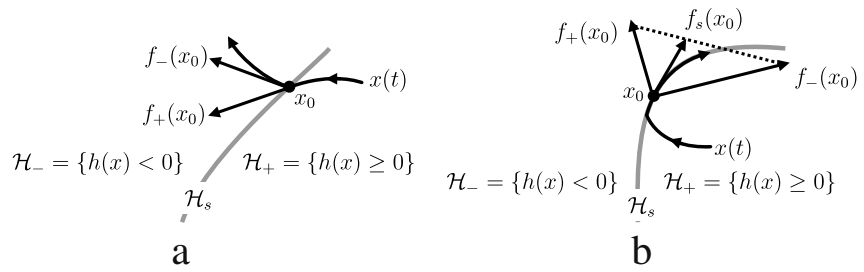

Fig. 1. A trajectory $x(t)$ through a point $x_{0}$ on the switching manifold $\mathscr{H}_{s}$ generically either (a) crosses the switching manifold $\mathscr{H}_{S}$ if the vector fields $f_{+}$and $f_{-}$both point in the same direction relative to $\mathscr{H}_{s}$, or (b) slides along $\mathscr{H}_{s}$ if $f_{+}$and $f_{-}$both point toward $\mathscr{H}_{s}$.

equilibrium or periodic orbit (and any of its bifurcations) observed in a simple model obtained by making quasi-static assumptions is also present when the fast dynamics is taken into account as long as the difference in time scale is sufficiently large. In general, in smooth dynamical systems any phenomenon that persists under regular perturbations (perturbations of the right-handside) also persists under stable singular perturbations. Fenichel's Theorem reduces hyperbolic singular perturbations to regular ones by proving the existence of a normally hyperbolic invariant manifold [5].

In order to find general statements how the dynamics of Filippov systems is affected by stable singular perturbations one has to study slow-fast systems of the form

$\dot{x}= \begin{cases}f_{-}(x, y, \varepsilon) & \text { if } h(x, y, \varepsilon)<0, \\ f_{+}(x, y, \varepsilon) & \text { if } h(x, y, \varepsilon) \geq 0,\end{cases}$

$\varepsilon \dot{y}=g(x, y, \varepsilon)$.

In system (3) and (4) $\varepsilon$ measures the difference in the time scales between the evolution of the slow variable $x$ and the evolution of the fast variable $y$. We assume that for $\varepsilon=0$ the (then algebraic) equation (4) can be solved for $y$ for all $x$, and that this solution $y_{0}(x)$ satisfies the stability condition

$0>-c>\operatorname{Re} \operatorname{spec} \partial_{2} g\left(x, y_{0}(x), 0\right)$

with a uniform decay rate $c$ (subscript 2 in the differential operator indicates differentiation with respect to the second argument). Condition (5) means that $y_{0}(x)$ is a locally exponentially stable equilibrium of the fast subsystem (4) if we treat the variable $x$ in (4) as a parameter and set $\varepsilon$ to 0 in the right-hand-side of (4).

Let us assume that the quasi-static approximation (called the reduced system from now)

$\dot{x}= \begin{cases}f_{-}\left(x, y_{0}(x), 0\right) & \text { if } h\left(x, y_{0}(x), 0\right)<0, \\ f_{+}\left(x, y_{0}(x), 0\right) & \text { if } h\left(x, y_{0}(x), 0\right) \geq 0\end{cases}$

has a stable periodic orbit $x(t)$ which is switching in $x_{0}=x\left(t_{0}\right)$ from the subdomain $\left\{x: h\left(x, y_{0}(x), 0\right) \geq 0\right\}$ to sliding inside the manifold $\left\{x: h\left(x, y_{0}(x), 0\right)=0\right\}$ (as shown in Fig. 1(b)). What happens to this periodic orbit if we include the singular perturbation effects by changing $\varepsilon$ to a positive value?

The references [6,7] studied this question under the simplifying assumption that the switching decision does not depend on the fast variable $y$ (that is, $\partial_{2} h=0$ in (3), (4)) and proved that stable periodic orbits persist when $\varepsilon$ becomes positive but they acquire small boundary layers after switching. In practice, it is often difficult to check or guarantee the condition $\partial_{2} h=0$. For example, a switch in an electronic circuit may depend on the voltage potential at a node $j$ which is only a function of neighbouring node potentials, which (let us assume) are all slow variables. However, taking into account a small parasitic capacitance (of order $\varepsilon$ ) affecting node $j$ will change its voltage potential into a fast variable, thus, making the switching decision dependent on a fast variable. The same effect occurs more generally in modelling: one often studies the simple model $(\varepsilon=0)$ only, and it is impossible to tell if switching decisions depend on fast variables for any of the possibly significant singular perturbations without studying the more complex model $(\varepsilon>0)$. This means that the assumption $\partial_{2} h=0$ of [6,7] is mathematically convenient because it allows one to prove persistence of stable periodic orbits but it does not cover all cases of practical interest.

Our paper studies the problem how the dynamics near a periodic orbit changes under a stable singular perturbation if the switching decision depends also on fast variables: $\partial_{2} h \neq 0$ in (3). It turns out that there are qualitative changes if we increase $\varepsilon$ from 0 to a positive value. Possible scenarios are, for example, a periodadding cascade on a parameter range of order $\varepsilon$, or small-scale chaos (also of order $\varepsilon$ ) around a periodic orbit that is exponentially stable for $\varepsilon=0$ but is uniformly unstable for all $\varepsilon>0$.

The paper is outlined as follows. In Section 2 we construct a simple example that shows how arbitrarily small stable singular perturbations can effect qualitative changes. From Section 3 onward we focus on the case of periodic orbits with a short region of sliding: this occurs close to so-called grazing-sliding bifurcations. First, in Section 3 we present a numerical observation for a minimal $(2+1)$-dimensional example based on the Hopf normal form. The example shows that, depending on the geometry of the problem, sliding may persist or not. In Section 4 we formulate a (still rather crude) general result about the persistence or destruction of sliding near grazing. Section 5 presents the $\varepsilon$-expansions of the local return maps for the minimal Hopf example. Finally in Section 6 we draw some initial conclusions and speculate along which lines we hope to generalise our results beyond the minimal example.

\section{Simple example: Destabilisation of pseudo-equilibria}

Let us start with the simplest possible example of a Filippov slow-fast system. This example demonstrates the mechanism by which stable singular perturbations can destroy stable sliding in Filippov systems. Consider the system

$\dot{x}=-\operatorname{sign}[\theta x+(1-\theta) y]$

$\varepsilon \dot{y}=x-y$.

In the quasi-static limit $\varepsilon=0$ system (7) and (8) has the pseudoequilibrium $x=y=0$ which is exponentially stable (even attracting in finite time). We recall that by a pseudo-equilibrium we mean an equilibrium of (7) and (8) that lies on the switching surface (a pseudo-equilibrium is typically not an equilibrium of any of the two flows near the surface). Consider the dynamics of the system (7) and (8) in the neighbourhood of this pseudoequilibrium for non-zero $\varepsilon$.

Let us express ( 7 ) and (8) in the general form given by (1), where

$f_{-}\left(\begin{array}{l}x \\ y\end{array}\right)=\left[\begin{array}{c}1 \\ \frac{1}{\varepsilon}(x-y)\end{array}\right], \quad f_{+}\left(\begin{array}{l}x \\ y\end{array}\right)=\left[\begin{array}{c}-1 \\ \frac{1}{\varepsilon}(x-y)\end{array}\right]$,

and the function $h(x, y)$ that defines the switching manifold $\mathscr{H}_{S}$ is given by

$h(x, y)=\theta x+(1-\theta) y$.

The vector normal to $\mathscr{H}_{s}$, is $\partial h=[\theta, 1-\theta]$. The conditions for the existence of an attracting sliding region read (see [4])

$\partial h f_{-}>0$ and $\partial h f_{+}<0$,

which gives for our example

$\theta+\frac{1-\theta}{\varepsilon}(x-y)>0, \quad-\theta+\frac{1-\theta}{\varepsilon}(x-y)<0$.

A subset of the switching manifold such that the vector fields $f_{-}$ and $f_{+}$both point towards the switching surface along this subset 


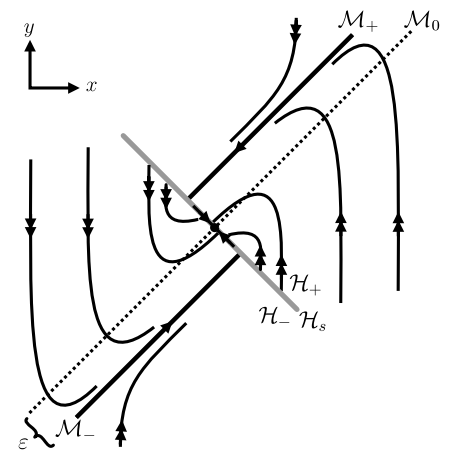

(a) $\theta>0$.

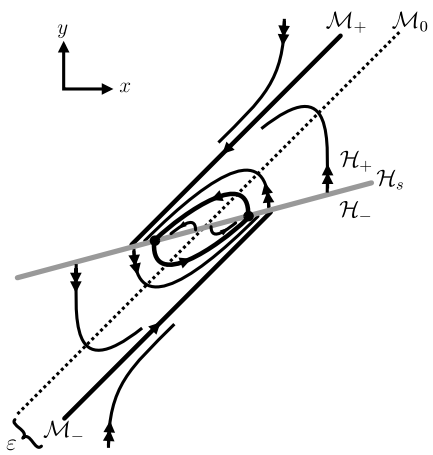

(b) $\theta<0$.

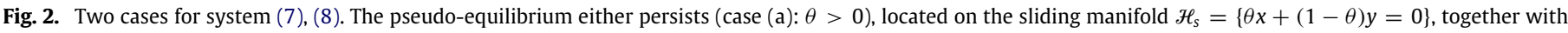

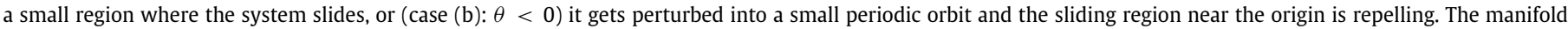

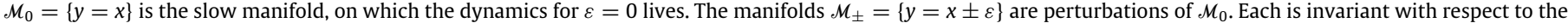
ODE on its side of the switching manifold, containing no rapidly decaying dynamics.

(see Fig. 1(b)) is called an attracting sliding region. A repelling sliding region is a subset of the switching manifold such that the vector fields $f_{-}$and $f_{+}$both point away from the switching surface along this subset.

For $\theta \neq 1$ the switching manifold $\mathscr{H}_{s}$ can be parameterised by $x$, thus, the condition (10) simplifies to $-\varepsilon \theta<x<\varepsilon \theta$ on $\mathscr{H}_{s}$. Therefore, if $\theta>0$ then (9) holds along a segment of the switching line $\mathscr{H}_{s}$ in a neighbourhood of the origin (see Fig. 2(a)) and the stable pseudo-equilibrium persists for positive $\varepsilon$ and stays at $x=y=0$. Moreover, in a sufficiently small neighbourhood of the origin the trajectories converge to this pseudo-equilibrium following the sliding flow.

If $\theta<0$ then (9) does not hold in the neighbourhood of the origin and there is no stable pseudo-equilibrium (there is a repelling sliding region near the origin) and instead we have a symmetric exponentially stable periodic orbit around $(0,0)$ switching back and forth between $f_{+}$and $f_{-}$. The point

$\left(x_{\text {switch }}, y_{\text {switch }}\right)=\left(\left(1-\theta^{-1}\right) y_{\text {switch }}, y_{\text {switch }}\right)$

where the periodic orbit switches from $f_{-}$to $f_{+}$is parameterised by (see also Fig. 2(b)):

$\theta=\frac{1}{2} \frac{T+2 \mathrm{e}^{-T}+\mathrm{e}^{-T} T-2}{1+\mathrm{e}^{-T}}, \quad y_{\text {switch }}=\varepsilon \theta \frac{\mathrm{e}^{-T}-1}{\mathrm{e}^{-T}+1}$

where $\varepsilon T$ is the travelling time that the orbit spends on each side. Thus, for $|\theta|$ of order $1(\theta<0)$ the amplitude and the period of the periodic orbit are of order $\varepsilon$; see Fig. 2(b) for the qualitative picture. Consequently, the dynamics of the perturbed system $(\varepsilon>0)$ are close to the dynamics of the unperturbed system $(\varepsilon=0)$ only in a very weak sense compared to the classical results of [5]: instead of a stable fixed point we have an exponentially stable periodic orbit of small amplitude. This chattering phenomenon is similar to what is observed when the switching occurs with a small delay [8-11]. Example (7) and (8) shows that chatter can also be introduced by fast dynamics even if the switching is perfectly instantaneous.

The example also shows that singular perturbations may alter the defining quantities (9) of the sliding region by order 1 uniformly for $\varepsilon \rightarrow 0$ and, thus, also change the dynamics qualitatively (in this example from exponentially stable pseudo-equilibrium to exponentially stable periodic orbit without sliding). This will be further highlighted in Section 5 where a periodic orbit in a singularly perturbed Filippov system is analysed in detail.

Fig. 2 shows the arrangement of invariant manifolds and the switching manifold for the example (7) and (8). For each of the two flows there exists an invariant manifold $\mathcal{M}_{ \pm}$consisting of all trajectories (in the 2D-example just one trajectory) without rapidly decaying part. These manifolds $\mathcal{M}_{ \pm}$are both $O(\varepsilon)$-perturbations of the so-called slow manifold $\mathcal{M}_{0}$, defined by setting $\varepsilon=0$ in the equation (8) for the fast dynamics. In the example the manifolds are $\mathcal{M}_{ \pm}=\{y=x \pm \varepsilon\}$ and $\mathcal{M}_{0}=\{x=y\}$. Every trajectory that spends a time of order 1 in the subdomain $\mathscr{H}_{+}=\{(x, y): h(x, y) \geq 0\}$ ends up exponentially close to the invariant manifold $\mathcal{M}_{+}$: its end point has a distance of order $\exp (-c / \varepsilon)$ from $\mathcal{M}_{+}$(similarly for $\mathcal{M}_{-}$in $\left.\mathscr{H}_{-}=\{(x, y): h(x, y)<0\}\right)$. The example shows that the invariant manifolds $\mathcal{M}_{+}$and $\mathcal{M}_{-}$typically differ by a term of order $\varepsilon$. This implies that any trajectory that switches back and forth between $\mathscr{H}_{+}$and $\mathscr{H}_{-}$shows a small boundary layer immediately after switching: after switching the trajectory has a short time interval during which it relaxes to the invariant manifold of the other flow. These small boundary layers occur also after switching to sliding and have been studied already in $[12,6,7]$. In example (7) for $\theta<0$ both parts of the periodic orbit are still in the boundary layer.

\section{Grazing-sliding in singularly perturbed Filippov systems - illustration}

In order to understand the effect of stable singular perturbations on periodic orbits with sliding we focus on periodic orbits with a short sliding segment. One common scenario for periodic orbits with short sliding segments is the so-called grazing-sliding event; see [4] for a classification of discontinuity-induced bifurcations. This phenomenon is a codimension-one event, which can be observed generically if the system depends on one additional parameter.

\subsection{Grazing-sliding - Illustration for minimal example}

A minimal example for the grazing-sliding event, the Hopf normal form for $f_{+}$combined with a constant vector field for $f_{-}$, is

$\dot{x}= \begin{cases}{\left[\begin{array}{l}\mu x_{1}-\omega x_{2}-\left(x_{1}^{2}+x_{2}^{2}\right) x_{1} \\ \omega x_{1}+\mu x_{2}-\left(x_{1}^{2}+x_{2}^{2}\right) x_{2}\end{array}\right]} & \text { if } x_{1} \geq-1 \\ {\left[\begin{array}{l}1 \\ 0\end{array}\right]} & \text { if } x_{1}<-1,\end{cases}$

where we keep $\omega \neq 0$ fixed and vary $\mu$ (which is always greater than 0 ) from below 1 to above 1 (see Fig. 3 ). The switching line for this example is

$h^{0}(x)=x_{1}+1$,

and the flow in $\mathscr{H}_{+}=\left\{h^{0} \geq 0\right\}$ has a unique stable periodic orbit as shown in Fig. 3(a). In fact, the flow in $\mathscr{H}_{+}$can be decomposed 


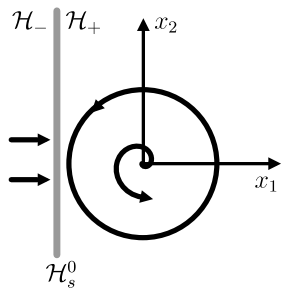

(a) $\mu<1$

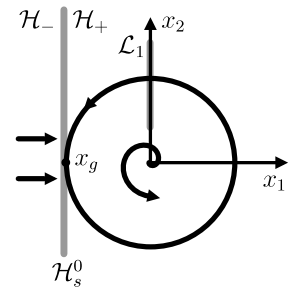

(b) $\mu=1$.

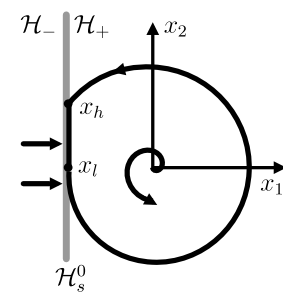

(c) $\mu>1$.

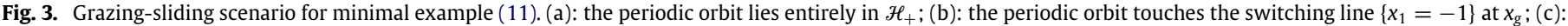
the periodic orbit has a small sliding segment from $x_{h}$ to $x_{l}$. The line $\mathcal{L}_{1}$ is the Poincaré section chosen for the return map $M_{1}$ in ( 12 ).

into a pair of uncoupled equations for the polar coordinates $r$ and $\varphi$ of $x \in \mathbb{R}^{2}(x=r \cdot(\cos \varphi, \sin \varphi))$ :

$\dot{r}=\mu r-r^{3}, \quad \dot{\varphi}=\omega$,

and the periodic orbit is a circle around the origin corresponding to $r=\sqrt{\mu}, \varphi=\omega t$. This periodic orbit is also a periodic orbit of the Filippov system (11) for $\mu<1$. When we change $\mu$ to values larger than 1 the periodic orbit of the Filippov system (11) acquires a sliding segment starting from some point $x_{h}=\left[-1, x_{h, 2}\right]^{T}\left(x_{2, h}>\right.$ $0)$ and ending at some point $x_{l}=\left[-1, x_{l, 2}\right]^{T}\left(x_{l, 2}<0\right.$ is defined by the condition $\partial h f_{+}\left(x_{l}\right)=0$ ), making the overall orbit piecewise smooth (shown in Fig. 3(c)): it has a corner at $x_{h}$ and its second derivative is discontinuous at $x_{l}$. The periodic orbit is grazing the switching manifold $\mathscr{H}_{s}^{0}=\left\{h^{0}=0\right\}$ for $\mu=1$ as shown in Fig. 3(b) in a point $x_{g}$, which satisfies several conditions simultaneously:

(1) The point $x_{g}$ lies on the stable periodic orbit of the flow in $\mathscr{H}_{+}$ and on the switching manifold $\mathscr{H}_{\mathrm{s}}^{0}$.

(2) The flow in $\mathscr{H}_{+}$is quadratically tangent to the switching manifold ( $\dot{h}=0$ and $\ddot{h}>0$ in $x_{g}$ along trajectories following $\left.f_{+}\right)$, and

(3) the flow in $\mathscr{H}_{-}$points toward the switching manifold in $x_{g}$.

The dynamics near the periodic orbit for $\mu \approx 1$ is described completely by its local return map (also called Poincaré map). This map is defined by following the flow from a small cross-section transversal to the periodic orbit back to itself. We choose the local return map $M_{\mu}$ to the section $\mathcal{L}_{1}=\left\{x: x_{1}=0, x_{2}>0\right\}$ (see Fig. $3(\mathrm{~b})$ ), which has for $\mu=1$ the form

$M_{1} x_{2}=1+ \begin{cases}\mathrm{e}^{-4 \pi / \omega}\left[x_{2}-1\right]+O\left(\left|x_{2}-1\right|^{2}\right) & \text { if } x_{2}<1 \\ 0 & \text { if } x_{2} \geq 1 .\end{cases}$

Thus, the map $M_{1}$ is piecewise asymptotically linear in its fixed point $x_{2}=1$ corresponding to the periodic orbit. For $\mu>1$ the map $M_{\mu}$ is constant near its fixed point. In summary, in system (11) the only effect of the grazing under variation of $\mu$ is that the periodic orbit changes its shape, and that its Floquet multiplier jumps from $\exp (-4 \pi \mu / \omega)$ to 0 at the grazing parameter $\mu=1$. The periodic orbit is stable for all $\mu \approx 1$.

\subsection{Singular perturbation of grazing-sliding - Observations for minimal example}

Now let us consider a stable singular perturbation of system (11). We will couple (11) with a one-dimensional stable fast subsystem for a fast variable $y$ where the coupling also occurs in the switching function. Thus we obtain

$\dot{x}= \begin{cases}{\left[\begin{array}{l}\mu x_{1}-\omega x_{2}-\left(x_{1}^{2}+x_{2}^{2}\right) x_{1} \\ \omega x_{1}+\mu x_{2}-\left(x_{1}^{2}+x_{2}^{2}\right) x_{2}\end{array}\right]} & \text { if } \theta x_{1}+(1-\theta) y \geq-1 \\ {\left[\begin{array}{l}1 \\ 0\end{array}\right]} & \text { if } \theta x_{1}+(1-\theta) y<-1\end{cases}$

$\varepsilon \dot{y}=\varepsilon\left[\mu x_{1}-\omega x_{2}-\left(x_{1}^{2}+x_{2}^{2}\right) x_{1}\right]+x_{1}-y$.
The quasi-static approximation replaces $y$ by $y_{0}(x, \mu)=x_{1}(\varepsilon=0$ in (14)). Thus, the reduced model is (11) which is identical with the slow part (13) of the singularly perturbed system except that the switching function depends now in part on the fast variable $y$ for $\theta \neq 1$. Namely

$h(x, y, \varepsilon)=\theta x_{1}+(1-\theta) y+1$.

The question is: how does the perturbation from the reduced system (11) to the slow-fast system (13) and (14) affect the local return map $M_{1}$ of the periodic orbit at $\mu=1$ ? For $\mu=1$ the grazing periodic orbit drawn in Fig. 3(b) exists also in the slow-fast system: its grazing point is now $p_{g}=(-1,0,-1)$, and on the orbit the fast variable is given by $y(t)=x_{1}(t)$. We make the following observation (see also Fig. 4):

Observation 1 (Perturbation of Local Return Map Near Grazing). The perturbed return map for $\varepsilon>0$ is a one-dimensional return map $P$ to the line $\mathscr{L}_{1}=\left\{(x, y) ; x_{1}=y=1\right\}$ if we ignore terms of order $\exp (-c / \varepsilon)$.

If $\theta<0$ then the map $P$ is discontinuous for $\varepsilon>0$, and the discontinuity is of size $O(\varepsilon)$.

If $\theta>0$ then the return map $P$ is continuous also for $\varepsilon>0$ but the slope near the point $p_{g}$ is of order 1 uniformly for $\varepsilon \rightarrow 0$ and $x_{2} \geq 1$.

Fig. 4 shows how the return map $M_{1}$ of the grazing periodic orbit is perturbed for positive $\varepsilon$, and $\theta<0$ (Fig. 4(a)) and $\theta>0$ (Fig. 4(b)). The grazing point $p_{g}$ corresponds to $x_{2}=\mu=1$. For $x_{2}<1$ the return map $P$ is identical to $M_{1}$. We observe that for $\theta<0$ the return map becomes discontinuous at $x_{2}=1$ and that the size of the jump is of order $\varepsilon$. Also, the limiting slope for $x_{2}>1$ appears to be $-\infty$. For $\theta>0$ we note that the limiting slope for $x_{2} \rightarrow+1$ is not of order $\varepsilon$. The slope is negative and of order 1 . For comparison, the map $M_{1}$ is identical to 1 for $x_{2}>1$. The change of shape of the graph of the return map from $M_{1}$ to $P$ has consequences for the dynamics near the grazing orbit. For $\theta<0$ the period-one orbit disappears as $\mu$ passes through 1 from below and we observe an inverted period-adding cascade [13]. For $\theta>0$ the period-one orbit persists but may change its stability depending on the slope of the graph in Fig. 4(b) for $x_{2} \rightarrow+1$. The local attractor (if any) for $\mu>1$ also depends on this limiting slope (see [4] for a recent textbook overview of possible scenarios in piecewise locally linear maps).

\section{Singular perturbation of grazing-sliding events}

This section gives a criterion that helps to distinguish whether a given periodic orbit that grazes the switching surface in a general slow-fast Filippov system undergoes a bifurcation as shown in Fig. 4(a) (that is, the local return map is discontinuous) or if we have a grazing-sliding case shown in Fig. 4(b) (the local return map is continuous). Let us assume that the reduced Filippov system (6) depends on a parameter $\mu$, and that (6) has a family of periodic orbits $x(t ; \mu)$. We assume without loss of generality that the 


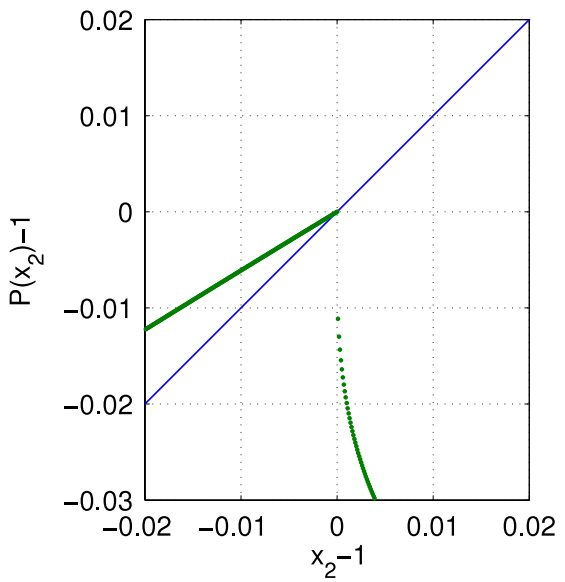

(a) $\theta=-0.5$.

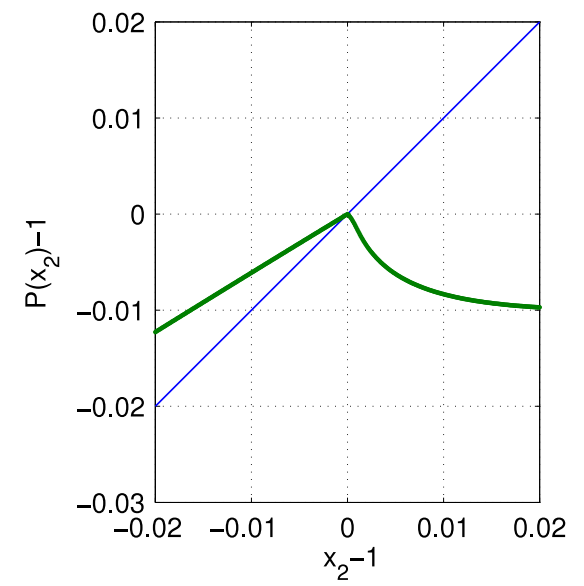

(b) $\theta=0.5$.

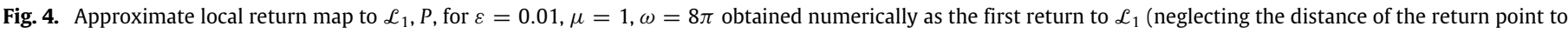
the slow invariant manifold $\mathcal{M}_{+}=\left\{(x, y) ; x_{1}=y\right\}$ of the flow in $\left.\mathscr{H}_{+}\right)$. Intersections with the diagonal indicate fixed points corresponding to period-one orbits.

periodic orbit $x(t ; \mu)$ has no sliding segment near $t=0$ for $\mu<0$, and that it lies entirely in the region $\mathscr{H}_{+}=\left\{x: h\left(x, y_{0}(x), 0\right) \geq 0\right\}$ locally near $t=0$ (see Fig. 3(a)). The precise characterisation for a grazing-sliding event for the family $x(t ; \mu)$ at a parameter value $\mu_{0}$ ( $\mu_{0}=0$ without loss of generality) can be given by using the function $\chi_{0}:(t, \mu) \mapsto h\left(x(t ; \mu), y_{0}(x(t ; \mu)), 0\right)$. The function $\chi_{0}$ gives the value of $h$ depending on time along the periodic orbit. The family $x(t ; \mu)$ grazes at $\mu=0$ if there exists a time $t_{0}$ near 0 such that

(1) $\chi_{0}\left(t_{0}, 0\right)=0\left(x\left(t_{0} ; 0\right)\right.$ is on the switching manifold $\left.\mathscr{H}_{s}\right)$,

(2) $\partial_{1} \chi_{0}\left(t_{0}, 0\right)=0$ (the orbit grazes $\mathscr{H}_{s}$ in $\left.t_{0}\right)$,

(3) $\partial_{1}^{2} \chi_{0}\left(t_{0}, 0\right)>0$ (the grazing is quadratic),

(4) $\partial_{2} \chi_{0}\left(t_{0}, 0\right)<0$ (the parameter $\mu$ unfolds the grazing),

(5) $\left[\partial_{1} h+\partial_{2} h \partial_{2} g^{-1} \partial_{1} g\right] f_{-}>0$ in $\left(x\left(t_{0} ; 0\right), y_{0}\left(x\left(t_{0} ; 0\right)\right), 0\right)$ (the reduced vector field in $\mathscr{H}_{-}=\left\{x: h\left(x, y_{0}(x), 0\right)<0\right\}$ points toward switching manifold).

(The functions $h, f_{ \pm}$and $y_{0}$ may all depend on $\mu$ in this list.) Note that this is a codimension-one event because we have two equality conditions (1) and (2) and can adjust the time $t_{0}$. The conditions 1-5 guarantee that the periodic orbit and the switching manifold interact as shown in Fig. 3(b). See also [4] for a detailed discussion of grazing-sliding events for periodic orbits.

Furthermore, let us assume that the periodic orbit $x(t ; \mu)$ is entirely in $\mathscr{H}_{+}$for $\mu<0$ (not only locally near $t_{0}$ ). Then the standard singular perturbation theory of [5] guarantees that for sufficiently small $\varepsilon>0$ the full system (3), (4) also has a family of periodic orbits $(x, y)(t ; \mu, \varepsilon)$ which depends smoothly on $\mu$ up to some parameter value $\mu_{\varepsilon}=O(\varepsilon)$ where it grazes quadratically at a point $\left(x_{\varepsilon}, y_{\varepsilon}\right)=(x, y)\left(t_{0} ; \mu_{\varepsilon}, \varepsilon\right)$. We can keep the time point of the grazing equal to $t_{0}$ independent of $\varepsilon$ without loss of generality by shifting the time in the family of periodic orbits because the slowfast system (3), (4) is autonomous. This means that the function $\chi_{\varepsilon}:(t, \mu) \mapsto h(x(t ; \mu, \varepsilon), y(t ; \mu, \varepsilon), \varepsilon)$ also satisfies the first four conditions $1-4$ for $\mu=\mu_{\varepsilon}$ and $t_{0}$. This is a simple persistence argument because only the smooth vector field $f_{+}$is involved. However, in general the equivalent of condition 5 (that the vector field on the other side points toward the switching manifold) is not necessarily satisfied for the full system (3), (4) even though it is satisfied for the reduced system.

The following lemma states how this affects the local return map of the periodic orbit at $\mu_{\varepsilon}$ :

Lemma 2 (Grazing-Sliding of Periodic Orbits). Suppose that the reduced system (6) depends on a parameter $\mu$ and has a grazingsliding periodic orbit, lying entirely in $\mathscr{H}_{+}$, and satisfying the conditions $1-5$ for the parameter value $\mu=0$ at $x_{0}=x\left(t_{0} ; 0\right)$.
Then for sufficiently small $\varepsilon>0$ the family of periodic orbits in the full system (3) and (4) also grazes for a nearby parameter value $\mu_{\varepsilon}=O(\varepsilon)$ and we have two generic cases.

Case 1 If $\partial_{1} h f_{-}-\partial_{1} h f_{+}>0$ in $\left(x_{0}, y_{0}\left(x_{0}\right), 0\right)$ then for sufficiently small $\varepsilon>0$ the local return map of the grazing periodic orbit is continuous (and piecewise smooth).

Case 2 If $\partial_{1} h f_{-}-\partial_{1} h f_{+}<0$ in $\left(x_{0}, y_{0}\left(x_{0}\right), 0\right)$ then for sufficiently small $\varepsilon>0$ the local return map of the grazing periodic orbit is discontinuous in the fixed point corresponding to the grazing periodic orbit.

In our set-up the local return map does not depend on the choice of the cross-section as long as the cross-section is not taken at $t_{0}$ (return maps to different cross-sections are equivalent and the coordinate change is a diffeomorphism obtained by following the flow in $\mathscr{H}_{+}$).

As the minimal example (6) shows, a typical feature of the grazing-sliding event is that the local return map along the periodic orbit is piecewise smooth in its fixed point corresponding to the periodic orbit $x(\cdot ; \mu)$ for the parameter $\mu=\mu_{\varepsilon}$ at which grazing occurs. More precisely, the return map is smooth (with a uniformly bounded derivative) everywhere except along a manifold containing the fixed point. At this manifold the map is only (Lipschitz) continuous. The lemma states that in the second case the return map of the grazing orbit in the singularly perturbed system develops a discontinuity for positive $\varepsilon$ at this manifold. The appearance of a discontinuity is guaranteed because we assume that the grazing orbit lies entirely in one domain, always following the flow $\left(f_{+}, g\right)$ (see Appendix A for details). In Section 5 we show that for the minimal example (13) and (14) the size of the discontinuity can be expected to be of order $\varepsilon$.

The quantity distinguishing case 1 and case 2 can be evaluated without knowledge of the right-hand-side $g$ of the fast dynamical subsystem (4). This means that one can check if sliding persists under stable singular perturbations of a term $y_{0}(x)$ in the reduced model without having to know how this perturbation looks like.

The two cases are distinguished by checking if the vector field in the domain $\mathscr{H}_{-}=\{(x, y): h(x, y, \varepsilon)<0\}$ points toward the switching surface $\mathscr{H}_{s}$ near the grazing point. It turns out that in the second case the reason for the discontinuity of the return map is the presence of a small repelling sliding region. For the first case the sliding condition is satisfied such that sliding occurs and the general theory developed in [4] (Theorem 8.1) applies. The proof of Lemma 2, given in Appendix A, simply has to check this sliding condition. Theorem 8.1 of [4] can also be used to approximate the one-sided derivatives of the local return map of the full system (3), 


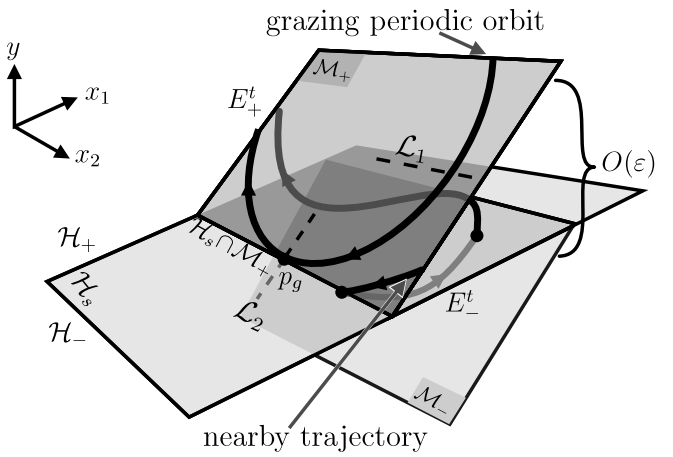

Fig. 5. Illustration of the manifolds near the grazing point $p_{g}$ for the slow-fast example (15), (16) for $\varepsilon>0, \theta<0$ and $\mu=1$ (zoom-in near the grazing point $p_{g}$ of the periodic orbit). The grazing periodic orbit lies entirely in $\mathscr{H}_{+}$(and, thus, in $\mathcal{M}_{+}$). The other invariant manifold $\mathcal{M}_{-}$(for $\mathscr{H}_{-}$) has a distance of $O(\varepsilon)$ from $\mathcal{M}_{+}$ The illustration shows the grazing periodic orbit and a typical (for $\theta<0$ ) switching trajectory near the periodic orbit, switching from $E_{+}$to $E_{-}$, back to $E_{+}$, and then re-approaching $\mathcal{M}_{+}$.

(4) for the sliding case (case 1) of Lemma 2. We show in Section 5 for the minimal example (13), (14) that the limit for $\varepsilon \rightarrow 0$ for these one-sided derivatives can be different from the one-sided derivatives obtained for the reduced model.

Lemma 2 is rather crude: a more detailed analysis is required to find out how the return map of the grazing periodic orbit actually looks like and how its features (for example, the size of the discontinuity or the one-sided derivatives) depend on $\varepsilon$. This analysis is technical for a general system such as (3) and (4), in particular if the dimension of the fast variable $y$ is larger than 1 . Thus, we construct the $\varepsilon$-expansions for the return map only for the minimal example (13) and (14). The example allows us to address both cases of Lemma 2 by varying its system parameter $\theta$. As long as the fast variable is one-dimensional, the generalisation to arbitrary singularly perturbed systems with a grazing-sliding periodic orbit is straightforward.

\section{Expansion of return maps for the minimal example}

Let us return to the minimal example

$\dot{x}= \begin{cases}{\left[\begin{array}{l}\mu x_{1}-\omega x_{2}-\left(x_{1}^{2}+x_{2}^{2}\right) x_{1} \\ \omega x_{1}+\mu x_{2}-\left(x_{1}^{2}+x_{2}^{2}\right) x_{2}\end{array}\right]} & \text { if } \theta x_{1}+(1-\theta) y \geq-1 \\ {\left[\begin{array}{l}1 \\ 0\end{array}\right]} & \text { if } \theta x_{1}+(1-\theta) y<-1\end{cases}$

$\varepsilon \dot{y}=\varepsilon\left[\mu x_{1}-\omega x_{2}-\left(x_{1}^{2}+x_{2}^{2}\right) x_{1}\right]+x_{1}-y$

where $\omega>0, \theta<1$ and $\mu \approx 1$. We have chosen the righthand-side $g$ of the fast Eq. (16) such that for $\varepsilon>0$ the subspace $\mathcal{M}_{+}=\left\{(x, y): x_{1}=y\right\}$ is the exact slow invariant manifold of the flow $E_{+}^{t}$ (following $\dot{x}=f_{+}, \varepsilon \dot{y}=g$ ). That is, any trajectory will converge to $\mathcal{M}_{+}$with a convergence rate of order $\varepsilon^{-1}$ as long as it stays in the half space $\mathscr{H}_{+}=\left\{(x, y): \theta x_{1}+(1-\theta) y \geq-1\right\}$. The graph of the invariant manifold $\mathcal{M}_{+}$for all $\mu$ and $\varepsilon$ is given by

$y_{m,+}(x)=x_{1}$.

Since $f_{+}$does not depend on $y$ the stable fibres of the manifold $\mathcal{M}_{+}$are: $\mathcal{F}_{+}\left(x_{0}\right)=\left\{(x, y): x=x_{0}, y \in \mathbb{R}\right\}$ for all $x_{0} \in \mathbb{R}^{2}$ (all points within a stable fibre converge to each other with a rate $O\left(\varepsilon^{-1}\right)$ following flow $E_{+}^{t}$ forward in time). The graph of the slow invariant manifold $\mathcal{M}_{-}$of $E_{-}$(the flow following $\dot{x}=f_{-}, \varepsilon \dot{y}=g$ ) is not known analytically. Its expansion up to order $\varepsilon$ is

$$
\begin{aligned}
y_{m,-}(x, \mu, \varepsilon)= & x_{1}+\varepsilon\left[\mu x_{1}-\omega x_{2}-\left(x_{1}^{2}+x_{2}^{2}\right) x_{1}-1\right] \\
& +O\left(\varepsilon^{2}\right) .
\end{aligned}
$$

The expressions for the slow invariant manifolds for $E_{+}$and $E_{-}$, (17) and (18) differ from each other by a term of order $\varepsilon$; the difference is $\varepsilon+O\left(\varepsilon^{2}\right)$ for $x=(-1,0), y=-1, \mu=1$, thus, it is non-zero at the grazing point $p_{g}=(-1,0,-1)$ at $\mu=1$. Hence, we expect that any trajectory crossing the switching manifold will show a small boundary layer; see Fig. 5 for an illustration of the invariant manifolds $\mathcal{M}_{ \pm}$and the switching manifold $\mathscr{H}_{s}=\{(x, y)$ : $\theta x_{1}+(1-\theta) y=-1$ ) near the grazing point at $\mu=1$.

The switching manifold $\mathscr{H}_{s}$ for the example (15) and (16) is a two-dimensional plane. Its intersection with the invariant manifold $\mathcal{M}_{+}$is

$$
\mathscr{H}_{s} \cap \mathcal{M}_{+}=\left\{(x, y): x_{1}=y=-1\right\} \text {. }
$$

The two cases of Lemma 2 correspond to $\theta>0$ (Case 1 ) and $\theta<0$ (Case 2). They have to be treated differently and result in qualitatively different return maps as Fig. 4 shows. The return maps for both cases are superpositions of a smooth global return map (following $E_{+}$) and a correction in the vicinity of the grazing, the Poincaré-section discontinuity mapping (PDM, [4]). In the following we derive an expression for the return map to the section $\Sigma_{1}=$ $\left\{(x, y): x_{1}=0, x_{2}>0, y \in \mathbb{R}\right\}$ which is away from the grazing point (see Fig. 5 for $\mathcal{L}_{1}=\Sigma_{1} \cap \mathcal{M}_{+}$). More specifically we consider the local return map to a small neighbourhood $U \subset \Sigma_{1}$ of the point $(x, y)^{T}=(0,1,0)^{T}$, the fixed point at $\mu=1$ corresponding to the grazing periodic orbit. Any trajectory starting in $\mathcal{U}$ spends a time of approximately $\pi /(2 \omega)$ following $E_{+}$before it reaches the vicinity of the switching manifold $\mathscr{H}_{S}$ in a small neighbourhood $\mathcal{V}$ of the point $p_{g}=(-1,0,-1)^{T}$. During this time the difference between $y$ and $x_{1}$ (which is the distance to the slow invariant manifold $\mathcal{M}_{+}$) decays such that $\left|y-x_{1}\right| \sim \exp (-\pi /(2 \omega \varepsilon))$ when the trajectory reaches $\mathcal{V}$, which is beyond all orders of $\varepsilon$. A trajectory leaving $\mathcal{V}$ follows $E_{+}$for a time of approximately $3 \pi /(2 \omega)$ until it reaches $\Sigma_{1}$. Again, after this time the $y$-component and the $x_{1}$-component of the trajectory will be $\exp (-2 \pi /(3 \omega \varepsilon))$ close to each other. This means that the return map $P$ is a one-dimensional map from the line $\mathcal{L}_{1}=\Sigma_{1} \cap \mathcal{M}_{+}=\left\{(x, y): x_{1}=y=0, x_{2}>0\right\}$ back to itself if we ignore terms of order $\exp (-c / \varepsilon)$ where $c>0$ is of order 1 (the return line $\mathcal{L}_{1}$ is also indicated by a dashed line in Fig. 5). The overall return map $P$ is a composition of four maps. Calling the time derivative of $h$ with respect to each of the flows as $h_{ \pm}^{\prime}$, respectively,

$$
\begin{aligned}
h_{ \pm}^{\prime}(x, y) & =\left.\partial h(x, y) \frac{\mathrm{d}}{\mathrm{d} t} E_{ \pm}^{t}(x, y)\right|_{t=0} \\
& =\left[\partial_{1} h f_{ \pm}+\varepsilon^{-1} \partial_{2} h g\right](x, y),
\end{aligned}
$$

(dropping the argument $\varepsilon$ ) the maps $P_{1}$ to $P_{4}$ act as follows

(1) $P_{1}$, maps from the line $\mathscr{L}_{1}=\left\{x_{1}=y=0, x_{2}\right\} \cap \mathcal{U}$ to the curve $\mathcal{L}_{2}=\left\{x_{1}=y, h_{+}^{\prime}(x, y)=0\right\} \cap \mathcal{V}$ by following the flow $E_{+}$ within the slow invariant manifold $\mathcal{M}_{+}$(see Fig. 5),

(2) $P_{2}$, the Poincaré-section discontinuity mapping from $\mathcal{L}_{2}$ to the surface $\Sigma=\left\{(x, y): h_{+}^{\prime}(x, y)=0\right\} \cap \mathcal{V}$, which contains the curve $\mathcal{L}_{2}$,

(3) $P_{3}$, maps from $\Sigma$ back to $\mathcal{L}_{2}$, following the projection along the stable fibres of $E_{+}: P_{3}\left(x_{1}, x_{2}, y\right)=\left(x_{1}, x_{2}, x_{1}\right)$,

(4) $P_{4}$, maps from $\mathscr{L}_{2} \subset \mathcal{V}$ back to $\mathscr{L}_{1} \subset \mathcal{U}$ following $E_{+}$inside the slow invariant manifold $\mathcal{M}_{+}$.

The composition

$P=P_{4} \circ P_{3} \circ P_{2} \circ P_{1}$

maps the line $\mathscr{L}_{1}$ back onto itself and it is a one-dimensional approximation of the true two-dimensional return map near the grazing periodic orbit up to terms of order $\exp (-c / \varepsilon)$. The map $P$ is conjugate (up to the diffeomorphism $P_{1}$ ) to the map

$\left[P_{1} \circ P_{4}\right] \circ\left[P_{3} \circ P_{2}\right]=: P_{\text {glob }} \circ P_{\mathrm{DM}}$, 
which maps from $\mathcal{L}_{2}$ back to itself. The smooth map $P_{\text {glob }}=P_{1} \circ P_{4}$ : $\mathcal{L}_{2} \mapsto \mathcal{L}_{2}$ is the global return map around the periodic orbit following the smooth flow $E_{+}$. Thus, $P_{\text {glob }}$ has the fixed point

$x_{1, \text { glob }}=-\sqrt{\mu}$

corresponding to the stable periodic orbit of $E_{+}$. Its linearisation at the fixed point is stable and is identical to the non-trivial Floquet multiplier of the periodic orbit of $E_{+}$, which is $\exp (-4 \pi \mu / \omega)$. This means that the smooth global return map $P_{\text {glob }}=P_{1} \circ P_{4}$ is described to first order by

$$
\begin{aligned}
P_{\text {glob }}\left(x_{1}\right)= & -\sqrt{\mu}+\exp (-4 \pi \mu / \omega)\left[x_{1}-x_{1, \text { glob }}\right] \\
& +O\left(\left[x_{1}-x_{1, \text { glob }}\right]^{2}\right) .
\end{aligned}
$$

The other part of the return map $P$, the projected discontinuity mapping

$P_{\mathrm{DM}}=P_{3} \circ P_{2}$

depends on the sign of the parameter $\theta$ in the switching function $h$. For each sign of $\theta$ we study a range of $\mu, x$ and $y$ near the grazing point $(x, y)=p_{g}=(-1,0), \mu=1$. The size of the range depends on $\varepsilon$ : it is $O(\varepsilon)$ for $\theta<0$, and it is $O\left(\varepsilon^{2}\right)$ for $\theta>0$. We take this into account by coordinates corresponding to a zoom-in into the neighbourhood of the the grazing point $p_{g}$ (blowing up the small neighbourhood to size 1 ).

\section{Lemma 3 (Discontinuity Mappings).}

Case $\theta<0$ : Introducing the coordinates $\xi_{1}$ and $q$ by $x_{1}=-1+$ $\varepsilon \xi_{1}$ and $\mu=1+\varepsilon q$, the map $P_{\mathrm{DM}}$ has the form

$$
\begin{aligned}
& P_{\mathrm{DM}}\left(\xi_{1}\right) \\
& \quad=\left\{\begin{aligned}
\xi_{1} \quad \text { if } \xi_{1} \geq 0 \\
s_{0}(\theta)+\sqrt{\varepsilon}\left[\frac{\omega s_{0}(\theta)}{\theta+s_{0}(\theta)} \sqrt{-2 \xi_{1}}+\frac{1}{\omega}\left(-2 \xi_{1}\right)^{3 / 2}\right.
\end{aligned}\right] \\
& +O(\varepsilon) \quad \begin{array}{l}
\text { if } \xi_{1}<0 .
\end{array}
\end{aligned}
$$

Case $\theta>0$ : Introducing the coordinates $\xi_{1}$ and $q$ by $x_{1}=-1+$ $\varepsilon^{2} \xi_{1}$ and $\mu=1+\varepsilon^{2} q$, the map $P_{\mathrm{DM}}$ has the form

$$
P_{\mathrm{DM}}\left(\xi_{1}\right)= \begin{cases}\xi_{1} & \text { if } \xi_{1} \geq 0 \\ s\left(\theta, \omega, \xi_{1}, \varepsilon\right) \xi_{1} & \text { if } \xi_{1}<0\end{cases}
$$

where the graph $s\left(\theta, \omega, \xi_{1}, \varepsilon\right) \xi_{1}$ is parametrically defined by the sliding time $t_{s}>0$. The zero-order term can be represented as (See Fig. 6.)

$$
\begin{aligned}
& \xi_{1}=-\frac{\omega^{2} \theta^{2}}{2}\left[\frac{(1-\theta)\left[1-\exp \left(-t_{s} / \theta\right)\right]+t_{s}}{\theta+(1-\theta) \exp \left(-t_{s} / \theta\right)}\right]^{2} \\
& s\left(\theta, \omega, \xi_{1}, 0\right) \xi_{1}=\omega^{2}(1-\theta) \frac{\theta-\left[\theta+t_{s}\right] \exp \left(-t_{s} / \theta\right)}{\theta+(1-\theta) \exp \left(-t_{s} / \theta\right)} .
\end{aligned}
$$

The quantity $s_{0}(\theta)$ appearing in (21) is a uniformly positive constant (only depending on $\theta$ ). It is implicitly defined by the Eq. (B.15) in Appendix B.1 (see also graph of $s_{0}(\theta)$ in Fig. B.2). The remainder term $O(\varepsilon)$ in $(21)$ for $\xi_{1}<0$ may contain small corrections to the constant and square-root terms in $\xi_{1}$. However, the form (21) guarantees that for sufficiently small values of the singular perturbation parameter $\varepsilon$ the overall return map $P_{\text {glob }} \circ P_{\mathrm{DM}}$ changes from a piecewise linear map for the reduced model $(\varepsilon=0)$ to a discontinuous map for the full model $(\varepsilon>0)$. The size of the jump at the discontinuity is of order $\varepsilon$ in the original coordinates. Moreover, the slope of the map next to the discontinuity is infinity from one side. Fig. 4(b) shows a numerically computed graph of the approximate overall return map $P$ to $\mathcal{L}_{1}$ for $\theta=-0.5, \varepsilon=10^{-2}$ and $\mu=1$ near the critical value of the coordinate $x_{2}$. This graph neglects exponentially small terms (the numerical distance of the

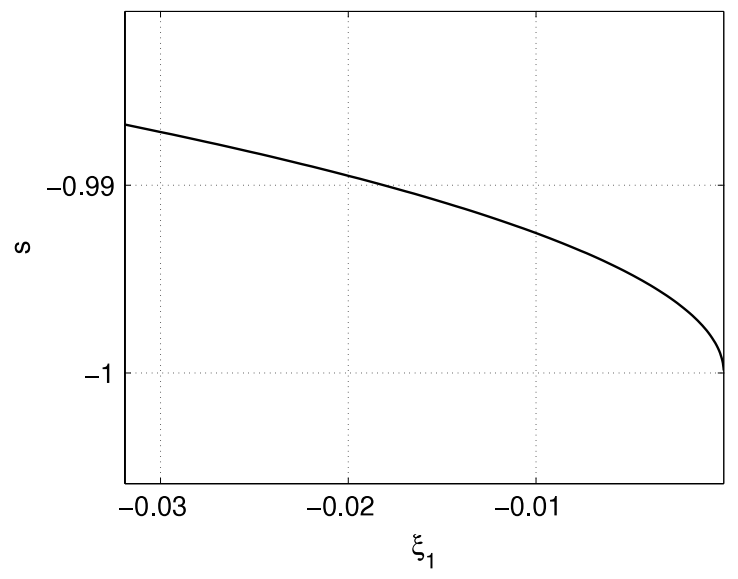

Fig. 6. Illustration of the implicitly defined graph $s(\theta, \omega, \cdot, 0)$, as defined by (23) (parameterised by $t_{s}, \omega=8 \pi, \theta=0.5$ ).

return value to the slow invariant manifold $\mathcal{M}_{+}$is of the order $10^{-10}$ at the return section). Appendix B. 1 gives the details of the derivation of the expansion of the map $P_{\mathrm{DM}}$ in (21).

In (22) the function $s$ is smooth in all arguments such that higher order terms do not destroy the continuity of the map $P_{\mathrm{DM}}$. Fig. 6 shows the graph of $s\left(0.5,8 \pi, \xi_{1}, 0\right)$. The value of $s$ at $\xi_{1}=0$ (that is, $\left.t_{s}=0\right)$ is $(\theta-1) / \theta$. Appendix B.2 gives the details of the derivation for the discontinuity mapping $P_{\mathrm{DM}}$ in (22). The change of the slope $s$ from its value at $\xi_{1}=0$ to slope 0 for $\xi_{1} \rightarrow-\infty$ (as predicted by the reduced model) occurs over a range of order $\varepsilon^{2}$ in the original coordinates after grazing. Fig. 4(a) shows the approximate overall return map $P$ to $\mathcal{L}_{1}$ for $\theta=0.5, \varepsilon=10^{-2}$ and $\mu=1$ near the critical value of the coordinate $x_{2}$.

\section{Conclusion}

Stable singular perturbations have a much stronger influence on the dynamics in Filippov systems than in smooth dynamical systems. We demonstrate that stable pseudo-equilibria and stable periodic orbits with sliding do not necessarily persist. We study periodic orbits with an infinitesimally small sliding segment, that is, close to a grazing-sliding bifurcation. We found two generic cases depending on the geometry: the local return map around the grazing periodic orbit develops a discontinuity if the condition on the existence of an attracting sliding region is violated. Otherwise, the continuity of the return map persists but the asymptotic slope may have a change of order 1 (uniform for $\varepsilon \rightarrow 0$ ).

The qualitative change of the local return map induces qualitative changes to the dynamics on a small scale. A piecewise discontinuous map with a square-root singularity of the slope on one side of the discontinuity, as occurs for $\theta<0$ in the minimal example, shows inverted period-adding cascades of periodic orbits if one varies the parameter $\mu$ through its critical value [13]. The parameter range where these cascades can be observed is of order $\varepsilon$ In the other case, $\theta>0$, the observed dynamics depends strongly on the one-sided derivative $s(\theta, 0,0)$ defined in Lemma 3. It can be chaotic if $s(\theta, 0,0)<-1$, which is possible for small $\theta$. Our analysis is valid on a scale of order $\varepsilon^{2}$ in phase space and parameter space.

The results of Lemma 3 can be generalised to higherdimensional slow-fast systems in a straightforward manner as long as the dimension of the fast subsystem is 1 . There are, however, some technical difficulties in generalising the expressions of Lemma 3 for higher dimensional fast subsystems $\left(y \in \mathbb{R}^{m}, m>1\right)$; a trajectory following the dynamics inside the stable fibres (following a linear stable ODE) may intersect the switching hyperplane several times. For the expressions (21) and (22) in Lemma 3 we 
exploited that this is not the case. In $\mathbb{R}^{1}$ every trajectory in a stable linear system approaches the origin in a monotone (increasing or decreasing) fashion, which is not true in $\mathbb{R}^{2}$ in the Euclidean norm. Furthermore, the Poincaré-section discontinuity mapping is only implicitly given as the root of a nonlinear equation even for the minimal example. In general, this implicit expression is determined by the intersection of a trajectory following a stable linear system with a hyperplane.

What are the consequences of the small-scale instabilities described in our paper in practical applications? They offer one explanation for discrepancies between predictions of a simple model and real observations: if the model is a smooth dynamical system and predicts stable periodic motion then observed noise around the predicted periodic motion in real observations is evidence for the presence of (unmodelled) inhomogeneous forcing. Fenichel's Theorem [5] guarantees that unmodelled strongly stable degrees of freedom (that is, a fast subsystem) cannot be the cause of this apparent noise. If the model is a Filippov system and sliding occurs in the predicted periodic motion then the fast subsystem can disturb the periodic motion in a way that it is no longer periodic (for example, chaotic, or periodic with a much higher period). Hence, if the model predicts sliding motion then apparent noise in the observation is not evidence for unmodelled inhomogeneous forcing.

The influence of fast dynamics also provides a mechanism for chatter (that is, rapid sequences of switches instead of sliding) which competes with other possible mechanisms. For example, switches in electronic circuits are known to be non-ideal [2]. Suppose that an electronic switch acts with a small delay $\tau$ but is otherwise ideal. Taking this delay into account one would predict similar effects as described in this paper [9-11]. Similarly, if the switches are implemented by fast actuators (with speed $1 / \tau$ as described in [6]) one would expect to observe chattering with a frequency of the order $1 / \tau$. However, if unmodelled capacities (or other stable degrees of freedom) give rise to fast stable subsystems with decay times $\varepsilon>\tau$ then the frequency of the chatter will be limited by $1 / \varepsilon$, which is smaller than $1 / \tau$.

Another open question is if mechanical systems with dry friction have a special structure that prevents singular perturbations from destroying sliding (the case $\theta<0$ in our examples). Singular perturbations in mechanical systems may certainly change the stability of sliding periodic orbits near grazing. This is also possible in the case corresponding to $\theta>0$ in our examples: sliding persists but at grazing the one-sided derivative of the grazing orbit has a change of order 1 due to the fast dynamics. We demonstrate this effect in Appendix $\mathrm{C}$ for the classical autonomous dry-friction oscillator.

\section{Acknowledgement}

Piotr Kowalczyk would like to acknowledge EPSRC grant EP/E050441/1.

\section{Appendix A. Proof of Lemma 2}

The assumptions of Lemma 2 imply that the system

$$
\begin{aligned}
& \dot{x}= \begin{cases}f_{-}(x, y, \mu, \varepsilon) & \text { if } h(x, y, \mu, \varepsilon)<0, \\
f_{+}(x, y, \mu, \varepsilon) & \text { if } h(x, y, \mu, \varepsilon) \geq 0\end{cases} \\
& \varepsilon \dot{y}=g(x, y, \mu, \varepsilon)
\end{aligned}
$$

has a family of periodic orbits $(x(t ; \mu, \varepsilon), y(t ; \mu, \varepsilon))$ that grazes the manifold $\mathscr{H}_{s}=\{h=0\}$ for all sufficiently small $\varepsilon>0$ : this follows from the fact that the reduced vector field $(\varepsilon=0)$ has a family of periodic orbits $(x(t ; \mu, 0), y(t ; \mu, 0))$ which grazes at $\mu=0$, and that the slow-fast system (A.1) and (A.2) on its slow invariant manifold $\mathcal{M}_{+}$in the domain $\mathscr{H}_{+}=\{h \geq 0\}$ is a regular perturbation of the reduced vector field. Without loss of generality we can (by reparameterising $\mu$ ) assume that the grazing occurs at $\mu=0$ for all $\varepsilon$, that the time at which grazing occurs on the orbit is $t=0$ for all $\varepsilon>0$, and that the point $(x(0,0, \varepsilon), y(0,0, \varepsilon))$ in which the orbit grazes is $(x, y)=(0,0)$ (by shifting time of the autonomous system and shifting the origin of the coordinate system). In our new coordinates the family $(x(t ; \mu, \varepsilon), y(t ; \mu, \varepsilon))$ is a family of periodic orbits, which depends smoothly on both parameters $(\mu, \varepsilon)$ in the parameter interval $\left[-\mu_{\max }, 0\right] \times\left[0, \varepsilon_{\max }\right]$ (both, $\mu_{\max }$, and $\varepsilon_{\max }$ are chosen sufficiently small). The periodic orbits are entirely inside $\mathscr{H}_{+}$and graze $\mathscr{H}_{s}$ quadratically in $(0,0)$ for $\mu=0$ (for all $\varepsilon \in\left[0, \varepsilon_{\max }\right]$ ).

This quadratic grazing condition means that the function

$\chi:(t, \mu, \varepsilon) \mapsto h(x(t ; \mu, \varepsilon), y(t ; \mu, \varepsilon), \mu, \varepsilon)$

(which is smooth on $\mathbb{R} \times\left[-\mu_{\max }, 0\right] \times\left[0, \varepsilon_{\max }\right]$ ) satisfies for all $\varepsilon \in\left[0, \varepsilon_{\max }\right]:$

(1) $\chi(0,0, \varepsilon)=0\left(\mathscr{H}_{s}\right.$ contains the origin for $\left.\mu=0\right)$

(2) $\partial_{1} \chi(0,0, \varepsilon)=0$ (the periodic orbit grazes $\mathscr{H}_{S}$ in the origin for $\mu=0)$

(3) $\partial_{1}^{2} \chi(0,0, \varepsilon)>0$ (the grazing is quadratic)

(4) $\partial_{2} \chi(0,0, \varepsilon)<0$ ( $\mu$ unfolds the grazing).

In order to find a local return map of the periodic orbit $(x(t, 0, \varepsilon), y(t, 0, \varepsilon))$ we have to choose an appropriate Poincaré cross section: the conditions 2 and 3 guarantee that the set

$\Sigma:=\left\{(x, y): \varepsilon \partial_{1} h f_{+}+\partial_{2} h g=0,\|(x, y)\|<\delta\right\}$

defines a smooth hypersurface near the origin, which intersects the periodic orbit and the switching manifold $\mathscr{H}_{S}$ transversally for all sufficiently small $\varepsilon$ and $\mu$. The set $\Sigma$ is the set of local minima of $h$ along trajectories of the flow $E_{+}$generated by the vector field $\left(f_{+}, g / \varepsilon\right)$. We define our Poincaré section as $\Sigma_{1}:=E_{+}^{-T(\mu, \varepsilon) / 2} \Sigma$ where $T(\mu, \varepsilon)$ is the period of the periodic orbit and $E_{+}^{t}$ is the time$t$ map of the flow $E_{+}$.

Theorem 8.1 in textbook [4] states that, if the vector field $\left(f_{-}, g / \varepsilon\right)$ points toward $\mathscr{H}_{s}(0, \varepsilon)$ in the origin then the local return map to $\Sigma_{1}$ is continuous and it can be written in the form

$P=E_{+}^{\Sigma \mapsto \Sigma_{1}} \circ P_{\mathrm{DM}} \circ E_{+}^{T(\mu, \varepsilon) / 2}$

where $E_{+}^{\Sigma \mapsto \Sigma_{1}}$ is the map obtained by following $E_{+}$from $\Sigma$ to its first intersection with $\Sigma_{1}$ (this is locally a diffeomorphism, the travelling time is close to $T(\mu, \varepsilon) / 2$ ). The map $P_{\text {DM }}$ maps $\Sigma$ back to itself and accounts for all effects due to the discontinuity (it is called the Poincaré discontinuity map in [4]). Whether the vector field $\left(f_{-}, g / \varepsilon\right)$ points toward or away from the switching manifold in the origin is determined by the sign of the function

$\sigma_{-}:(x, y, \mu, \varepsilon) \mapsto\left[\partial_{1} h f_{-}+\varepsilon^{-1} \partial_{2} h g\right](x, y, \mu, \varepsilon)$

in the point $(x, y, \mu, \varepsilon)=(0,0,0, \varepsilon)$. Due to condition 2 we know that the time derivative of $h$ when following $E_{+}$vanishes in $(0,0$, $0, \varepsilon)$ :

$\partial_{1} h f_{+}+\varepsilon^{-1} \partial_{2} h g=0$.

Thus, combining (A.3) and (A.4) we obtain that the quantity distinguishing the two cases in Lemma 2 is in fact

$\sigma_{-}(0,0,0, \varepsilon)=\partial_{1} h f_{-}-\partial_{1} h f_{+}$

That is, in case 1 the vector field $E_{-}$points toward the switching manifold $\mathscr{H}_{s}$ and we can apply Theorem 8.1 from [4], proving the claim of Lemma 2 for case 1 , whereas, in case $2, E_{-}$points away from the switching manifold $\mathscr{H}_{s}$. Theorem 8.1 from [4] also gives an asymptotic expression for the one-sided derivatives of the map $P_{\mathrm{DM}}$ in $(0,0)$. The example from Section 5 will show that the limit 
of these one-sided derivatives for $\varepsilon \rightarrow 0$ can be different from the one-sided derivative when one applies Theorem 8.1 from [4] to the reduced system $(\varepsilon=0)$.

It remains to be shown that in case 2 , when the vector field $\left(f_{-}, g / \varepsilon\right)$ points away from $\mathscr{H}_{s}$ in the origin for $\mu=0$, the return map to $\Sigma_{1}$ is discontinuous for $\varepsilon>0$. Let us define the sets

$H_{1}^{+}=E_{+}^{-T(\mu, \varepsilon) / 2}\{(x, y) \in \Sigma: h(x, y, \varepsilon) \geq \varepsilon\}$

$N=E_{+}^{\Sigma \mapsto \Sigma_{1}}\{(x, y) \in \Sigma: h(x, y, \varepsilon)<0\}$.

The set $H_{1}^{+}$consists of all points in the Poincaré section $\Sigma_{1}$ for which $P_{\mathrm{DM}}$ is the identity (that is, trajectories starting in $\mathrm{H}_{1}^{+}$do not switch to the flow $E_{-}$during the evaluation of the return map $P$ ). The set $N$ consists of points in $\Sigma_{1}$ that do not have a pre-image (they are images of a repelling sliding section under $E_{+}^{\sum_{\mapsto} \mapsto \Sigma_{1}}$ ).

The set $\mathrm{PH}_{1}^{+} \subset \Sigma_{1}$ can be written as

$P H_{1}^{+}=E_{+}^{\Sigma \mapsto \Sigma_{1}}\{(x, y) \in \Sigma: h(x, y, 0) \geq 0\}$

since $E_{+}^{\Sigma \mapsto \Sigma_{1}} \circ E_{+}^{T(\mu, \varepsilon) / 2}$ is the whole return map $P$ when $P_{\mathrm{DM}}$ is the identity. Thus, the set $P H_{1}^{+} \cup N$ is an image of the whole cross-section $\Sigma$ under the local diffeomorphism $E_{+}^{\Sigma \mapsto \Sigma_{1}}$. Hence, $\mathrm{PH}_{1}^{+} \cup N$ contains an open neighbourhood of the fixed point $p_{0} \in \Sigma_{1}$ corresponding to the grazing periodic orbit. Moreover, $P$ is a diffeomorphism between $H_{1}^{+}$and $\mathrm{PH}_{1}^{+}$. This implies that points from $\Sigma_{1} \backslash H_{1}^{+}$cannot be mapped into the neighbourhood of $p_{0}$ under $P$ (they can neither be mapped into $N$ nor into $\mathrm{PH}_{1}^{+}$). However, $p_{0}$ is on the boundary of $H_{1}^{+}$and, thus, it is in the closure of $\Sigma_{1} \backslash H_{1}^{+}$. Consequently, there exist points arbitrarily close to $p_{0}$ that do not get mapped into the neighborhood of $p_{0}$ under $P$, which proves that $P$ is discontinuous in case 2 .

We note that the proof of discontinuity in case 2 of Lemma 2 is not specific to slow-fast systems (we only use that the vector field $E_{-}$points away from the switching manifold at the grazing point $(0,0,0, \varepsilon))$ but it relies on the fact that the grazing orbit lies entirely in the domain governed by the flow $E_{+}$(that is why $P$ restricted to $H_{1}^{+}$is a diffeomorphism). In general, for example, if the grazing periodic orbit has sliding segments of positive length in other parts of the phase space, the return map may not be discontinuous in case 2 .

\section{Appendix B. The Poincaré discontinuity mappings of the mini- mal example in Section 5}

This appendix derives the dominant terms of the projected discontinuity mapping

$P_{\mathrm{DM}}=P_{3} \circ P_{2}$

as defined for the minimal example in Section 5. The map $P_{\mathrm{DM}}$ maps the line $\mathcal{L}_{2}=\left\{h_{+}^{\prime}(x, y)=0, x_{1}=y\right\}$ back to itself in the neighbourhood of the grazing point $p_{g}=(-1,0,-1)$. Orbits of $E_{+}$crossing $\mathcal{L}_{2}$ in a point with coordinate $x_{1} \geq-1$ do not switch to $E_{-}$or slide: by definition of $\mathcal{L}_{2}$ each orbit has its local minimum of $h$ exactly when it crosses $\mathcal{L}_{2}$, and $h$ is identical to $x_{1}+1$ on the invariant manifold $\mathcal{M}_{+}$of $E_{+}$. Thus, $\mathcal{L}_{2}$ can be parameterised by the coordinate $x_{1}$, and for $x_{1} \geq-1$ the map $P_{\mathrm{DM}}$ is the identity: $P_{\mathrm{DM}}\left(x_{1}\right)=x_{1}$. For $x_{1}<-1$ the map $P_{\mathrm{DM}}$ is itself again a composition of several maps:

$P_{\mathrm{DM}}=\Pi_{3} \circ P_{3} \circ \Pi_{2} \circ \Pi_{1}$

where

- $\Pi_{1}$ follows the flow $E_{+}$backward in time from $\mathcal{L}_{2}$ to the switching manifold $\mathscr{H}_{s}$, specifically, the line $\mathcal{L}_{3}=\left\{\tilde{x}_{1}=\tilde{y}=\right.$ $0\}$,

- the map $\Pi_{2}$ depends on the sign of the parameter $\theta$ :

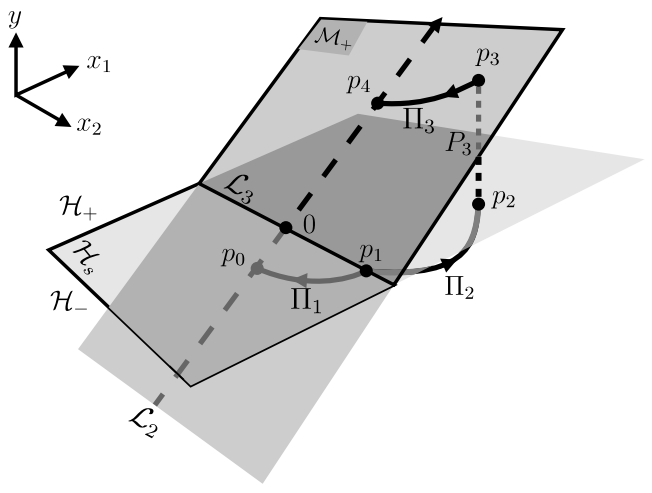

Fig. B.1. The Poincaré discontinuity map for the case $\theta<0$ near the point $p_{g}$ ( 0 in the rescaled coordindate system (B.2)).

$\theta<0: \Pi_{2}$ follows $E_{-}$from $\mathcal{L}_{3}$ forward in time until one hits the switching manifold $\mathscr{H}_{S}$ again;

$\theta>0: \Pi_{2}$ follows the sliding flow $E_{s}$, defined by (2), starting from $\mathcal{L}_{3}$ and staying in the switching surface $\mathscr{H}_{s}$ until the flow $E_{+}$becomes tangent to $\mathscr{H}_{s}$, that is, $h_{+}^{\prime}(x, y)$ becomes zero;

- $P_{3}$ projects along the stable fibre of $E_{+}$down onto the slow invariant manifold $\mathcal{M}_{+}: P_{3}\left(x_{1}, x_{2}, y\right)=\left(x_{1}, x_{2}, x_{1}\right)$.

- $\Pi_{3}$ follows $E_{+}$on $\mathcal{M}_{+}$(forward or backward in time) to $\mathscr{L}_{2}$.

We have swapped $P_{3}$ and $\Pi_{3}$ in (B.1), which is possible because $P_{3}$ and $\Pi_{3}$ commute.

\section{B.1. The case $\theta<0$}

(See Fig. B.1.) For $\theta<0$ the parameter range of interest is of order $\varepsilon$. Similarly, the scale of the dynamics transversal to the limit cycle (that is, the $x_{1}$ and the $y$ components) is of order $\varepsilon$. We zoom into the neighbourhood of the grazing point $p_{g}=(-1,0,-1)$ by introducing the new quantities $\xi, \psi$ and $q$ (which are rescalings of the coordinates $x, y$ and $\mu$ ):

$$
\begin{aligned}
& \varepsilon=\delta^{2} \quad x_{1}=-1+\delta^{2} \xi_{1} \\
& \mu=1+\delta^{2} q \quad x_{2}=\delta \xi_{2} \\
& y=-1+\delta^{2} \psi .
\end{aligned}
$$

Any point in the phase-parameter space with $\xi, \psi$ and $q$ of order 1 is in the vicinity of the grazing bifurcation. The scaling (B.2) squeezes the direction along the grazing periodic orbit by scaling it only by $\delta=\sqrt{\varepsilon}$. In the new coordinates the switching manifold $\mathscr{H}_{s}$ is $\left\{(\xi, \psi): \theta \xi_{1}+(1-\theta) \psi=0\right\}$. System (13) and (14) in the new coordinates has the form (with rescaled time $t_{\text {new }}=t_{\text {old }} / \delta$ and truncating terms of order $\delta^{3}$ ):

$\dot{\xi}=\left\{\begin{array}{c}{\left[\begin{array}{c}-\omega \xi_{2}+\left[\xi_{2}^{2}-2 \xi_{1}-q\right] \delta+O\left(\delta^{3}\right) \\ -\omega+\omega \delta^{2} \xi_{1}+O\left(\delta^{3}\right) \\ \text { if } \theta \xi_{1}+(1-\theta) \psi \geq 0\end{array}\right]} \\ {\left[\begin{array}{c}1 / \delta \\ 0\end{array}\right] \quad \text { if } \theta \xi_{1}+(1-\theta) \psi<0}\end{array}\right.$

$\delta \dot{\psi}=-\psi+\xi_{1}-\omega \xi_{2} \delta+\left[\xi_{2}^{2}-2 \xi_{1}-q\right] \delta^{2}+O\left(\delta^{4}\right)$.

We expand the quantities $h_{+}^{\prime}$ and $h_{-}^{\prime}$ in the new coordinates in $\delta$ (dropping $O\left(\delta^{3}\right)$ and substituting $\psi$ by $\theta \xi_{1} /(\theta-1)$ ):

$$
\begin{aligned}
\delta^{-1} h_{+}^{\prime}= & \xi_{1}-\omega \xi_{2} \delta+\left[\xi_{2}^{2}-q-2 \xi_{1}\right] \delta^{2}+O\left(\delta^{3}\right) \\
\delta^{-1} h_{-}^{\prime}= & \theta+\xi_{1}+(\theta-1) \omega \xi_{2} \delta \\
& +(1-\theta)\left[\xi_{2}^{2}-q-2 \xi_{1}\right] \delta^{2}+O\left(\delta^{3}\right)
\end{aligned}
$$


Thus, in the vicinity of the grazing point the vector field $E_{+}$points toward the switching manifold $\mathscr{H}_{S}$ if $h_{+}^{\prime}<0$, that is,

$\xi_{1}<\omega \xi_{2} \delta-\left[\xi_{2}^{2}-q\right] \delta^{2}+O\left(\delta^{3}\right)$,

and $E_{-}$points toward $\mathscr{H}_{S}$ if $h_{-}^{\prime}>0$, that is,

$$
\begin{aligned}
\xi_{1}> & -\theta+(1-\theta) \omega \xi_{2} \delta+(1-\theta)\left[q-\xi_{2}^{2}-2 \theta\right] \delta^{2} \\
& +O\left(\delta^{3}\right) .
\end{aligned}
$$

The curve $\mathcal{L}_{2}$ is nearly linear for $\xi$ of order 1 . It can be parameterised in the rescaled coordinates $\left[\xi_{1}, \xi_{2}, \psi\right]$ (see (B.2)) by the map

$L_{2}: \mathbb{R} \mapsto \mathbb{R}^{3}: \xi_{1} \mapsto\left[\xi_{1}, \eta\left(\xi_{1}\right), \xi_{1}\right]$

where the second component $\left(\xi_{2}\right)$ of $L_{2}$ is the locally unique solution $\xi_{2}$ of the equation

$h_{+}^{\prime}\left(\xi_{1}, \xi_{2}, \xi_{1}, q, \delta\right)=0$

depending on $\xi_{1}, q$ and $\delta$ (the grazing is quadratic, hence $h_{+}^{\prime}$ is monotone in $\xi_{1}$ ). More precisely, the solution $\xi_{2}$ of (B.9) can be represented as a graph $\eta\left(\xi_{1}\right)$ (dropping the dependence of $\eta$ on $q$ and $\delta$ ), and this graph can be expanded in $\delta$ :

$\eta\left(\xi_{1}\right)=-\frac{2 \xi_{1}+q}{\omega} \delta+\delta^{3} r\left(\xi_{1}, q, \delta\right)\left[\xi_{1}, q\right]$.

The remainder term of order $\delta^{3}$ in (B.10) is at most linear in $\xi_{1}$ and $q$ because $\eta(0)=0$ for $\xi_{1}=q=0$ and all $\delta$ (the grazing point $\xi_{1}=\xi_{2}=\psi=q=0$ satisfies (B.9) for all $\delta$ ).

The map $\Pi_{1}$ We expand the trajectory $\left(\xi_{1}(t), \xi_{2}(t), \psi(t)\right)$ of $E_{+}$ through a point

$p_{0}=\left(\xi_{1,0}, \eta\left(\xi_{1,0}\right), \xi_{1,0}\right) \in \mathcal{L}_{2}$

in time $\left(\psi(t)=\xi_{1}(t)\right.$ because $\left.p_{0} \in \mathcal{M}_{+}\right)$:

$$
\begin{aligned}
\xi(t)= & {\left[\begin{array}{c}
\xi_{1,0} \\
-\frac{2 \xi_{1,0}+q}{\omega} \delta+O\left(\delta^{3}\right)
\end{array}\right]+\left[\begin{array}{c}
0 \\
-\omega+O\left(\delta^{2}\right)
\end{array}\right] t } \\
& +\left[\begin{array}{c}
\frac{\omega^{2}}{2}+O\left(\delta^{2}\right) \\
O\left(\delta^{3}\right)
\end{array}\right] t^{2}+t^{3} O\left(\delta^{2}\right) .
\end{aligned}
$$

The coefficient in front of $t^{2}$ is non-zero in its first component because we have quadratic grazing. The first component of the coefficient in front of $t$ vanishes by choice of $\mathcal{L}_{2}$. Thus, for $\xi_{1,0}<0$ and $\left|\xi_{1,0}\right|$ of order 1 or less, the trajectory intersects the switching manifold $\left\{\xi_{1}=0\right\}$ at

$t= \pm \frac{\sqrt{-2 \xi_{1,0}}}{\omega}\left(1+O\left(\delta^{2}\right)\right)+O\left(\delta^{2}\right) \xi_{1,0}+O\left(\delta^{3}\right)$.

The map $\Pi_{1}$ is defined by following $E_{+}$backward in time. Thus, we choose the negative sign in (B.11). This gives rise to an intersection of the trajectory with the switching surface at a point $\left(0, \xi_{2,1}, 0\right)$ with the coordinate

$\xi_{2,1}=\sqrt{-2 \xi_{1,0}}\left(1+O\left(\delta^{2}\right)\right)-\frac{2 \xi_{1,0}+q}{\omega} \delta+O\left(\delta^{2}\right)$.

The first term of the sum in (B.12) contains all square-root terms of $\xi_{1,0}$. Consequently the map $\Pi_{1}$ maps

$\Pi_{1}: p_{0}=\left[\xi_{1,0}, \eta\left(\xi_{1,0}\right), \xi_{1,0}\right] \in \mathcal{L}_{2} \mapsto p_{1}=\left[0, \xi_{2,1}, 0\right] \in \mathcal{L}_{3}$

where $\xi_{2,1}$ is given by (B.12). The dominant term in the expansion of $p_{1}$ with respect to the coordinate $\xi_{1,0}$ of $p_{0}$ is a square root.

The map $\Pi_{2}$ For $\xi_{1,0}$ of order 1 (and, thus, $\xi_{2,1}$ of order 1 ) the flow $E_{-}$ points away from the switching surface $\mathscr{H}_{S}$ in $p_{1}$ because condition (B.7) is not satisfied in $p_{1}$ (because $\theta<0$ ). The initial point for

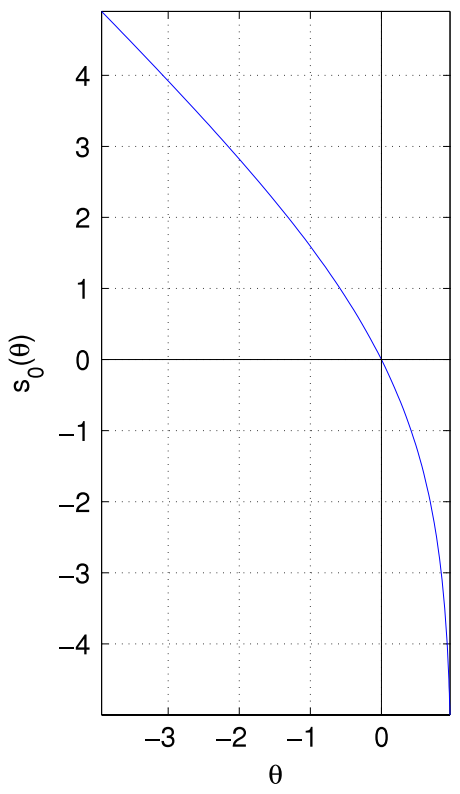

Fig. B.2. Implicitly given graph of $s_{0}(\theta)$.

the map $\Pi_{2}$ is $p_{1}=\left(0, \xi_{2,1}, 0\right)$. The trajectory $\left(\xi_{1}(s), \xi_{2}(s), \psi(s)\right)$ following $E_{-}$through this point satisfies

$$
\begin{aligned}
& \xi_{1}(s)=s \\
& \xi_{2}(s)=\xi_{2,1} \\
& \psi(s)=s+\exp (-s)-1+\omega \xi_{2,1}[\exp (-s)-1] \delta+O\left(\delta^{2}\right)
\end{aligned}
$$

where $s=t / \delta$ is the rescaled time. There is a unique time $s_{*}\left(\xi_{2,1}\right)$ $>0$ such that the trajectory hits the switching surface $\left\{\theta \xi_{1}+(1-\right.$ $\theta) \psi=0$ \} again. The expansion of $s_{*}\left(\xi_{2,1}\right)$ in $\delta$ is

$s_{*}\left(\xi_{2,1}\right)=s_{0}(\theta)+\delta \frac{\omega s_{0}(\theta)}{\theta+s_{0}(\theta)} \xi_{2,1}+O\left(\delta^{2}\right)$

and $s_{0}(\theta)$ is the unique positive solution of the equation

$(1-\theta) \exp \left(-s_{0}\right)-1+s_{0}+\theta=0$.

Fig. B.2 shows the graph of $s_{0}(\theta)$, which can be determined implicitly by expressing $\theta$ as a function of $s_{0}$. Since we assume that $|\theta|$ is of order 1 we cannot replace (B.14) by its expansion in $\theta$. The denominator in the coefficient for $\delta$ in (B.14) is positive because the solution $s_{0}$ of (B.15) is greater than $-\theta$ for all $\theta<0$. Consequently, $\Pi_{2}$ maps

$\Pi_{2}: p_{1}=\left(0, \xi_{2,1}, 0\right) \mapsto p_{2}=\left[s_{*}\left(\xi_{2,1}\right), \xi_{2,1}, \frac{-\theta s_{*}\left(\xi_{2,1}\right)}{1-\theta}\right]$.

For small $\delta$, and $\left|\xi_{1,2}\right|$ of order 1 or less, the sliding condition (B.6) is not satisfied for points in the image of $\Pi_{2}$ because $s_{0}(\theta)>0$ is of order 1 . This implies that the flow will switch to $E_{+}$. The projection $P_{3}$ of the image along the stable fibres of $E_{+}$

$$
\begin{gathered}
P_{3}: p_{2}=\left[s_{*}\left(\xi_{2,1}\right), \xi_{2,1}, \frac{-\theta s_{*}\left(\xi_{2,1}\right)}{1-\theta}\right] \\
\mapsto p_{3}=\left[s_{*}\left(\xi_{2,1}\right), \xi_{2,1}, s_{*}\left(\xi_{2,1}\right)\right]
\end{gathered}
$$

maps into the slow invariant manifold $\mathcal{M}_{+}$of $E_{+}$and away from the switching manifold because $0<-\theta /(1-\theta)<1$ and $s_{*}\left(\xi_{2,1}\right)>0$. Thus, the point $p_{3}$ is also in the half space $\mathscr{H}_{+}=\left\{(\xi, \psi): \theta \xi_{1}+\right.$ $(1-\theta) \psi>0\}$.

The map $\Pi_{3}$ The map $\Pi_{3}$ maps the point $p_{3}$ of the slow invariant manifold $\mathcal{M}_{+}$back to the Poincare section (more precisely its intersection with $\left.\mathcal{M}_{+}\right), \mathcal{L}_{2}$, by following $E_{+}$. This can be obtained 


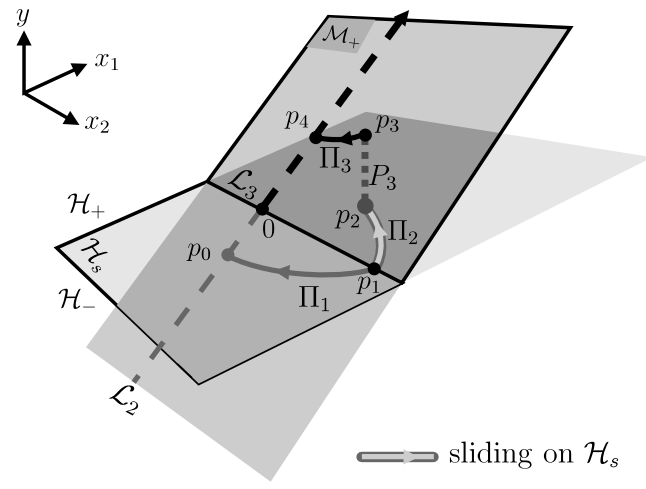

Fig. B.3. The Poincaré discontinuity map for the case $\theta>0$ near the point $p_{g}(0$ in the rescaled coordindate system (B.2)).

in the same manner as the map $\Pi_{1}$ : denote the components of $p_{3}$ by $\left[\xi_{1,3}, \xi_{2,3}, \psi_{3}\right]$ (where $\psi_{3}=\xi_{1,3}$ because $p_{3} \in \mathcal{M}_{+}$), and denote $\xi$-component of the trajectory $E_{+}^{t} p_{3}$ by $\left(\xi_{1}(t), \xi_{2}(t)\right)$. The time $t_{3}$ when the trajectory hits $\mathcal{L}_{2}$ has to satisfy the condition defining $\mathcal{L}_{2}$

$\eta\left(\xi_{1}\left(t_{3}\right)\right)=\xi_{2}\left(t_{3}\right)$

which defines $t_{3}$ and the point $p_{4}=\left(\xi_{1,4}, \xi_{2,4}, \psi_{4}\right)=\left(\xi_{1}\left(t_{3}\right)\right.$, $\left.\xi_{2}\left(t_{3}\right), \xi_{1}\left(t_{3}\right)\right)$ implicitly. We expand $\xi_{1}(t)$ and $\xi_{2}(t)$ with respect to time $t$ in 0 to second order in $t$ using (B.3) (all third-order terms have a coefficient of order $\delta^{3}$ ) and (B.10), and insert the expansion of $t_{3}$ in $\delta$,

$t_{3}=t_{3,0}+\delta t_{3,1}+O\left(\delta^{2}\right)$,

into (B.16) where $t_{3,0}$ and $t_{3,1}$ are the unknowns at zero and first order level. Solving (B.16) to first order in $\delta$ gives

$t_{3}=\frac{\xi_{2,3}}{\omega}+\frac{q+2 \xi_{1,3}}{\omega^{2}} \delta+O\left(\delta^{2}\right)$ and, thus,

$\xi_{1,4}=\xi_{1,3}+\frac{\xi_{2,3}^{3}}{\omega} \delta+O\left(\delta^{2}\right)$.

Consequently, the map $\Pi_{3}$ maps

$\Pi_{3}: p_{3}=\left[\xi_{1,3}, \xi_{2,3}, \xi_{1,3}\right] \mapsto p_{4}=\left[\xi_{1,4}, \eta\left(\xi_{1,4}\right), \xi_{1,4}\right] \in \mathcal{L}_{2}$

where $\xi_{1,4}$ is given (B.18).

The PDM Combining the expressions (B.12), (B.14) and (B.18) we obtain the composition $P_{\mathrm{DM}}=\Pi_{3} \circ P_{3} \circ \Pi_{2} \circ \Pi_{1}$ :

$P_{\mathrm{DM}}\left(\xi_{1}\right)=\left\{\begin{array}{l}\xi_{1} \quad \text { if } \xi_{1} \geq 0 \\ s_{0}(\theta)+\delta\left[\frac{\omega s_{0}(\theta)}{\theta+s_{0}(\theta)} \sqrt{-2 \xi_{1}}+\frac{\left(-2 \xi_{1}\right)^{3 / 2}}{\omega}\right] \\ \quad+O\left(\delta^{2}\right) \quad \text { if } \xi_{1}<0 .\end{array}\right.$

We have expressed the map $P_{\mathrm{DM}}$ as a map for the coordinate $\xi_{1}$ of the point on the curve $\mathcal{L}_{2}$. Given the coordinate $\xi_{1}$, the other coordinates of a point of $\mathcal{L}_{2}$ are $\xi_{2}=\eta\left(\xi_{1}\right)$ and $\psi=\xi_{1}$ where the expansion of $\eta$ is given in (B.10). The expression for $s_{0}(\theta)$ is given implicitly by (B.15). The remainder term $O\left(\delta^{2}\right)$ for $\xi_{1}<0$ may contain small corrections to the constant and square-root terms in $\xi_{1}$. However, the form (B.20) guarantees that for sufficiently small $\delta$ (and, thus, singular perturbation parameter $\varepsilon$ ) the overall return map $P_{g} \circ P_{\mathrm{DM}}$ changes from a piecewise linear map for the reduced model $(\varepsilon=0)$ to a discontinuous map for the full model $(\varepsilon>0)$. The size of the jump at the discontinuity is of order $\varepsilon$ in the original coordinates. Moreover, the slope of the map next to the discontinuity is infinity from one side.

\section{B.2. The case $\theta>0$}

(See Fig. B.3.) For $\theta>0$ the assumptions behind the validity for the asymptotic form of the PDM near a grazing-sliding orbit are satisfied for the singularly perturbed system [4]. The flow $E_{+}$is grazing the switching surface $\mathscr{H}_{s}=\left\{\theta x_{1}+(1-\theta) y=0\right\}$ quadratically in the point $p_{g}=[-1,0,-1]^{T}$. The other flow $E_{-}$points toward the switching surface near the grazing point $p_{g}$ of $E_{+}$. Thus, according to Theorem 8.1 of [4] we have a generic grazing-sliding bifurcation. In general, the zero-time discontinuity map $P_{Z}(Z D M)$ is piecewise asymptotically linear, having the form (ignoring higherorder terms in $v$ )

$P_{Z}\left(p_{g}+v\right)=p_{g}+ \begin{cases}v & \text { if } \partial h v \geq 0, \\ v-\frac{F_{-}-F_{+}}{\partial h F_{-}} \partial h v & \text { if } \partial h v<0\end{cases}$

where $v \in \mathbb{R}^{3}$ is small, $p_{g}$ is the grazing point, $\partial h$ is the normal of the switching surface, and $F_{ \pm}=\left(f_{ \pm}, g / \varepsilon\right)$ is the derivative of $E_{ \pm}$in $p_{g}$. In our example we have $p_{g}=[-1,0,-1]^{T}, \partial h=[\theta, 0,1-\theta]$, $F_{+}=[0,-\omega, 0]^{T}$ and $F_{-}=[1,0,0]^{T}$ at parameter $\mu=1$. Thus, at $\mu=1$ the linear approximation of the ZDM, $P_{Z}$, for points in $\mathcal{M}_{+}$, the invariant manifold of $E_{+}$, is

$P_{\mathrm{Z}}\left[\begin{array}{l}x_{1} \\ x_{2} \\ x_{1}\end{array}\right]= \begin{cases}{\left[\begin{array}{l}x_{1} \\ x_{2} \\ x_{1}\end{array}\right]} & \text { if } x_{1} \geq-1, \\ {\left[\begin{array}{c}\left(1-\theta^{-1}\right)\left(x_{1}+1\right)-1 \\ x_{2}-\omega \theta^{-1}\left(x_{1}+1\right) \\ x_{1}\end{array}\right]} & \text { if } x_{1}<-1 .\end{cases}$

The map $P_{Z}$ can be converted to the projected PDM $P_{\mathrm{DM}}$ by applying $P_{Z}$ to points in $\mathcal{L}_{2}$, then applying the projection $P_{3}$ along the stable fibres of $E_{+}$, and finally applying the map $\Pi_{4}$, which is the same as for the case $\theta<0$, following $E_{+}$on its invariant manifold $\mathcal{M}_{+}$back to $\mathcal{L}_{2}$. Thus, ignoring terms of order $x_{1}^{3 / 2}$ or higher, the PDM maps the coordinate $x_{1}$ of a point in $\mathcal{L}_{2}$ as follows:

$P_{\mathrm{DM}}\left(x_{1}\right)= \begin{cases}x_{1} & \text { if } x_{1} \geq-1 \\ {\left[1-\theta^{-1}\right]\left(x_{1}+1\right)-1} & \text { if } x_{1}<-1\end{cases}$

The slope is different for $x_{1}<-1$ from the slope of the reduced model $\left(\varepsilon=0\right.$, where $P_{\mathrm{DM}}\left(x_{1}\right)=-1$ for $\left.x_{1}<-1\right)$ if $\theta \in(0,1)$. The difference in the slope is uniform for all small $\varepsilon>0$ (expression (B.23) does not depend on $\varepsilon$ ). For parameters $\mu$ close to 1 the slope of $P_{\mathrm{DM}}$ for $x_{1}<-1$ changes at most by a term of order $\mu-1$. The slope of $P_{\mathrm{DM}}$ for $x_{1}<-1$ is negative for $\theta \in(0,1)$ and can be arbitrarily large in modulus, thus, giving rise to a strong expansion for small $\theta$ (and, possibly, chaos) in a small neighbourhood of the grazing orbit.

The remainder of this section will show that the size of this neighbourhood where the slope of $P_{\mathrm{DM}}$ is negative and non-small is at least of order $\varepsilon^{2}$ (judging from Fig. 4(b) it is larger). In order to see this we have to obtain the expressions for the maps $\Pi_{1}, \Pi_{2}$ and $\Pi_{3}$ for this case as well. The maps $\Pi_{1}$ and $\Pi_{3}$ are identical to the case $\theta<0$ apart from a slightly differently scaled set of coordinates. The map $\Pi_{2}$ follows the sliding flow $E_{s}$ to the curve $\left\{h_{+}^{\prime}(x, y)=0, h(x, y)=0\right\}$ in the case $\theta>0$, and is, thus, different from the map $\Pi_{2}$ for the case $\theta<0$.

We zoom into the neighbourhood of the grazing point by introducing the new quantities $\xi, \psi$ and $q$ with a scaling $\varepsilon^{2}$ (different from the scaling for $\theta<0$ ):

$x_{1}=-1+\varepsilon^{2} \xi_{1}, \quad y=-1+\varepsilon^{2} \psi$,

$x_{2}=\varepsilon \xi_{2}, \quad \mu=1+\varepsilon^{2} q$.

Similarly as for $\theta<0$, any point in the phase-parameter space with $\xi$, $\psi$ and $q$ of order 1 is in the vicinity of the grazing bifurcation. The 
switching manifold $\mathscr{H}_{s}$ is $\left\{(\xi, \psi): \theta \xi_{1}+(1-\theta) \psi=0\right\}$. System (13), (14) in the rescaled coordinates has the form (with rescaled time $t_{\text {new }}=t_{\text {old }} / \varepsilon$ and truncating terms of order $\varepsilon^{3}$ ):

$\dot{\xi}=\left\{\begin{array}{c}{\left[\begin{array}{c}-\omega \xi_{2}+\left[\xi_{2}^{2}-2 \xi_{1}-q\right] \varepsilon+O\left(\varepsilon^{3}\right) \\ -\omega+\omega \varepsilon^{2} \xi_{1}+O\left(\varepsilon^{3}\right) \\ \text { if } \theta \xi_{1}+(1-\theta) \psi \geq 0 \\ {\left[\begin{array}{c}1 / \varepsilon \\ 0\end{array}\right] \quad \text { if } \theta \xi_{1}+(1-\theta) \psi<0}\end{array}\right]}\end{array}\right.$

$\dot{\psi}=-\psi+\xi_{1}-\omega \xi_{2}+\left[\xi_{2}^{2}-2 \xi_{1}-q\right] \varepsilon+O\left(\varepsilon^{3}\right)$.

For system (B.25), (B.26) each trajectory can spend at most a time span of order 1 in any bounded ball (of size of order 1 ) in the $(\xi, \psi)$-coordinates. The expansions of the quantities $h_{+}^{\prime}$ and $h_{-}^{\prime}$ (determining where the system is sliding) in $\varepsilon$ are

$\varepsilon^{-2} h_{+}^{\prime}=\xi_{1}-\omega \xi_{2}+\left[\xi_{2}^{2}-q-2 \xi_{1}\right] \varepsilon+O\left(\varepsilon^{3}\right)$

$\varepsilon^{-2} h_{-}^{\prime}=\varepsilon^{-1} \theta+\xi_{1}+(\theta-1) \omega \xi_{2}+O(\varepsilon)$.

As the expression for $h_{-}^{\prime}$ is always positive for $\xi, \psi$ and $q$ of order 1 the system slides on $\mathscr{H}_{s}$ whenever $h_{+}^{\prime}<0$.

The expressions for the curve $\mathcal{L}_{2}$ and the maps $\Pi_{1}$ and $\Pi_{3}$ are identical to (B.8), (B.10), (B.12), (B.18) and (B.19). The only modification is the differently scaled set of coordinates (B.24). The effect of this scaling is that we have to replace $\delta$ by $\varepsilon$ in all equations from (B.9) to (B.12) and in (B.18), leaving all expressions identical otherwise. Thus, $\Pi_{1}$ and $\Pi_{3}$ map

$\Pi_{1}: p_{0}=\left[\xi_{1,0}, \eta\left(\xi_{1,0}\right), \xi_{1,0}\right] \in \mathcal{L}_{2} \mapsto p_{1}=\left[0, \xi_{2,1}, 0\right] \in \mathcal{L}_{3}$

$\Pi_{3}: p_{3}=\left[\xi_{1,3}, \xi_{2,3}, \xi_{1,3}\right] \mapsto p_{4}=\left[\xi_{1,4}, \eta\left(\xi_{1,4}\right), \xi_{1,4}\right] \in \mathcal{L}_{2}$

where $\eta, \xi_{2,1}$ and $\xi_{1,4}$ are given by

$\eta\left(\xi_{1}\right)=-\frac{2 \xi_{1}+q}{\omega} \varepsilon+\varepsilon^{3} r\left(\xi_{1}, q, \varepsilon\right)\left[\xi_{1}, q\right]$,

$\xi_{2,1}=\sqrt{-2 \xi_{1,0}}\left(1+O\left(\varepsilon^{2}\right)\right)-\frac{2 \xi_{1,0}+q}{\omega} \varepsilon+O\left(\varepsilon^{2}\right)$,

$\xi_{1,4}=\xi_{1,3}+\frac{\xi_{2,3}^{3}}{\omega} \varepsilon+O\left(\varepsilon^{2}\right)$.

The map $\Pi_{2}$ For $\xi_{1,0}$ (and, thus, $\xi_{2,1}$ ) of order 1 the flow $E_{-}$points toward the switching surface $\mathscr{H}_{s}$. In $p_{1}$ the flow $E_{+}$points toward the switching surface, too (as $h_{+}^{\prime}<0$ in $p_{1}$ ), such that $\Pi_{2}$ is defined by following the sliding flow $E_{s}^{t}$ until $h_{+}^{\prime}$ (given by (B.27)) becomes zero. The sliding flow $E_{s}^{t}$ is invariant on the switching manifold $\mathscr{H}_{s}$. It follows an ODE for $\xi$ ( $\psi$ is given by $\left.\psi=-\theta /(1-\theta) \xi_{1}\right)$. The right-hand-side of the ODE can be expanded in $\varepsilon$ as

$\dot{\xi}_{1}=-\frac{1}{\theta}\left[\xi_{1}-\omega \xi_{2}(1-\theta)\right]+O(\varepsilon)$

$\dot{\xi}_{2}=-\omega+O(\varepsilon)$.

The initial condition is $\left(0, \xi_{2,1}\right)$ (where $\left.\xi_{2,1}>0\right)$. The time $t$ that the system follows (B.28) until $h_{+}^{\prime}$ becomes zero is of order 1 such that the trajectory $\xi(t)$ is a perturbation of order $\varepsilon$ of

$$
\begin{aligned}
& \xi_{1}(t)=\omega(1-\theta)\left([1-\exp (-t / \theta)]\left(\theta \omega+\xi_{2,1}\right)-\omega t\right) \\
& \xi_{2}(t)=\xi_{2,1}-\omega t .
\end{aligned}
$$

We insert the trajectory (B.29) into the expansion (B.27) for $h_{+}^{\prime}$ to find the unique positive time $t_{2}$ for which $\varepsilon^{-2} h_{+}^{\prime}$ in (B.27) becomes zero in a point

$p_{2}=\left(\xi_{1,2}, \xi_{2,2}, \frac{\theta}{\theta-1} \xi_{1,2}\right)$.
This time $t_{2}$, the time for which for the system follows the sliding flow (B.28), is given implicitly to zero order in $\varepsilon$ by the equation

$\xi_{2,1}=\omega \theta \frac{(1-\theta)\left[1-\exp \left(-t_{2} / \theta\right)\right]+t_{2}}{\theta+(1-\theta) \exp \left(-t_{2} / \theta\right)}$.

The right-hand-side in (B.30) is uniformly monotone increasing in $t_{2}$, and it is zero for $t_{2}=0$. Thus, (B.30) can be solved for $t_{2}$. In combination with the zero-order approximations

$\xi_{1,2}=\omega^{2}(1-\theta) \frac{\theta-\left[\theta+t_{2}\right] \exp \left(-t_{2} / \theta\right)}{\theta+(1-\theta) \exp \left(-t_{2} / \theta\right)}$

$\xi_{2,2}=\xi_{1,2} / \omega$

for $\xi_{1,2}$ and $\xi_{2,2}$ one obtains well defined graphs for $\xi_{1,2}\left(\xi_{2,1}\right)$ and $\xi_{2,2}\left(\xi_{2,1}\right)$. As monotonicity persists under small perturbations these graphs remain well-defined also if we take all orders of $\varepsilon$ into account.

When we combine the graph $\xi_{1,2}\left(\xi_{2,1}\right)$ with the maps $\Pi_{1}$, the projection $P_{3}$ and the map $\Pi_{3}$ we obtain an implicit expression for the graph of the $P_{\mathrm{DM}}$ for $\xi_{1,0}<0$, parameterised by the sliding time $t_{2} \in \mathbb{R}^{+}$:

$\xi_{1,0}=-\frac{\omega^{2} \theta^{2}}{2}\left[\frac{(1-\theta)\left[1-\exp \left(-t_{2} / \theta\right)\right]+t_{2}}{\theta+(1-\theta) \exp \left(-t_{2} / \theta\right)}\right]^{2}$

$\xi_{1,4}=\omega^{2}(1-\theta) \frac{\theta-\left[\theta+t_{2}\right] \exp \left(-t_{2} / \theta\right)}{\theta+(1-\theta) \exp \left(-t_{2} / \theta\right)}$.

Its derivative at $\xi_{1,0}=0$ (that is, $t_{2}=0$ ) is $(\theta-1) / \theta$. This is in line with the piecewise linearisation (B.23), and gives evidence that $\varepsilon^{2}$ is really the smallest present scale.

\section{Appendix C. Mechanics example - Autonomous dry-friction oscillator}

We demonstrate the practical consequences of Lemma 2 for a simple mechanical system: the autonomous dry-friction oscillator as shown in Fig. C.1(a), a block of mass $M$ attached to a rigid wall via a spring of stiffness $K$ and slipping (or sticking) on a belt moving with velocity $v$ toward the wall. This setup is a caricature of the mechanics of, for example, violins or door squeal. A simple model for the dry-friction oscillator is

$\dot{x}_{1}=x_{2}$

$\dot{x}_{2}=-\frac{K}{M} x_{1}-\frac{1}{M} F_{c}\left(x_{2}-v\right)$

where $x_{1}$ is the position of the block, $x_{2}$ is its velocity and $F_{c}$ is the friction force exerted on the block by the belt and acting against the relative velocity. The switching between vector fields is hidden in the form of the friction force $F_{c}$, which is the sum of a smooth nonlinear function and a term $-f_{0} \operatorname{sign} h$ where $h$ is $h(x)=v-x_{2}$ for (C.1). See Fig. C.1(b) for a typical model of $F_{c}$. Fig. C.1(c) shows the bifurcation diagram predicted by model (C.1) (and qualitatively confirmed in experiments in [14]), showing that the equilibrium $x=\left(-F_{c}(-v) / K, 0\right)$ loses its stability in a subcritical Hopf bifurcation at $\mathrm{H}$ and that slip-stick oscillations exist and are stable up to the belt velocity at point GS. The slip-stick oscillations correspond to periodic orbits with sliding segment (physically corresponding to sticking: the block has zero velocity relative to the belt for a while). At GS the slip-stick oscillations lose their stability (and cease to exist) in a grazing-sliding bifurcation as introduced in Section 4 and discussed for the simple minimal example in Section 3.1 (except that the orbits lying entirely in $\mathscr{H}_{+}$ are unstable). Model (C.1) can be affected by singular perturbation in various ways. One possibility is that the velocity $v$ is not perfectly attained as a direct control parameter but instead a controlled motor is aiming to achieve a certain belt velocity $v_{0}$. This would imply 

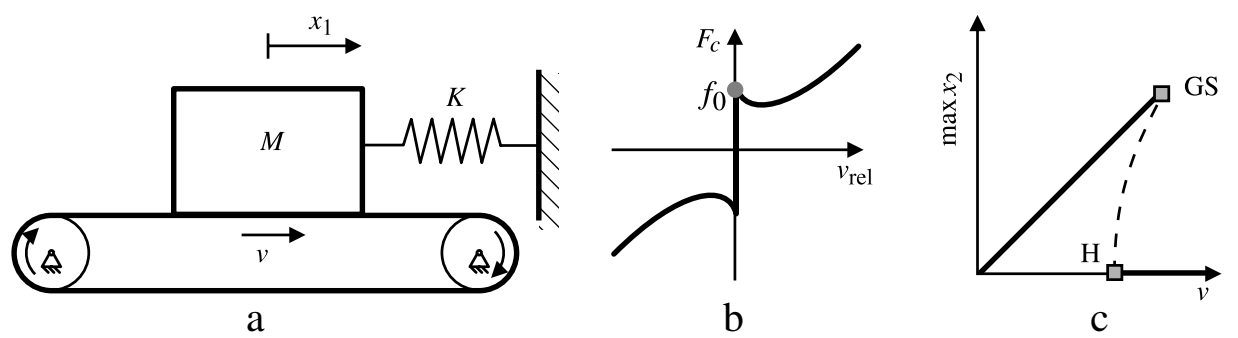

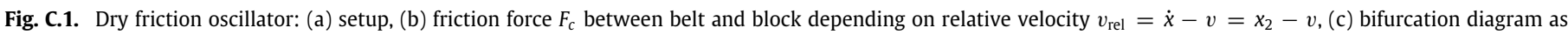
predicted by simple model (C.1) with a grazing-sliding bifurcation at GS.

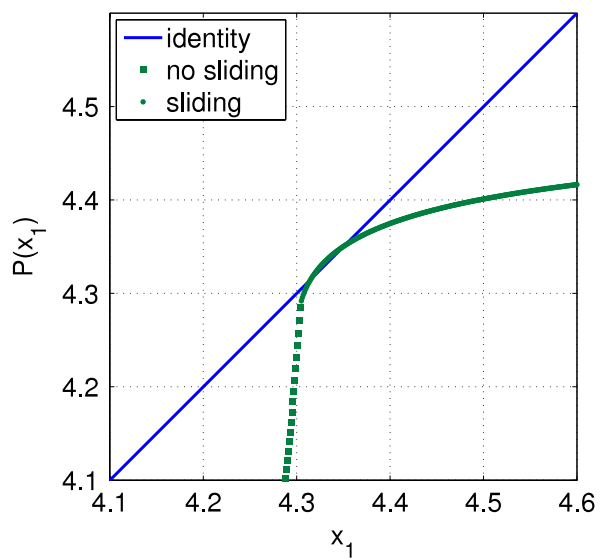

(a) $\varepsilon=0.1, v_{0}=3.32$.

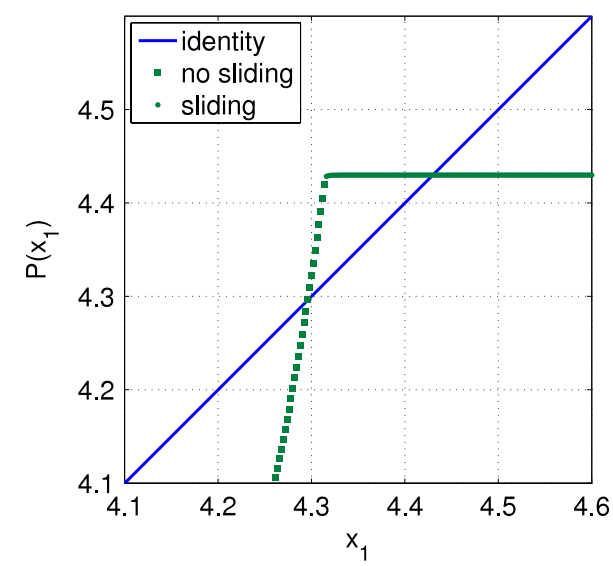

(b) $\varepsilon=0.01, v_{0}=3.1$

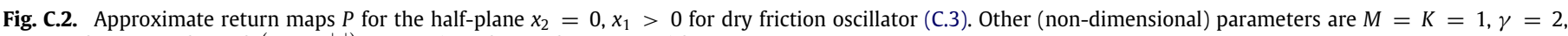
$F_{c}(v)=f_{0} \operatorname{sign} v+f_{v} v+f_{c}\left(1-\mathrm{e}^{-|v|}\right) \operatorname{sign} v$ where $f_{0}=2, f_{v}=0.3$, and $f_{c}=-0.5$.

that $v$ is determined as a dependent variable of a fast subsystem, for example

$M_{\text {belt }} \dot{v}=a\left[v_{0}-v\right]+F_{c}\left(x_{2}-v\right)$

where $a$ is the proportional control gain and $M_{\text {belt }}$ is the effective mass of the belt. If we introduce the variable $w=\left(M / M_{\text {belt }}\right) x_{2}+v$ and the parameter $\gamma=M / M_{\text {belt }}$, and treat $M_{\text {belt }} / a$ as the small parameter $\varepsilon$ then system (C.1), (C.2) is in the slow-fast form (3) and (4) as described in the introduction. Namely we have

$\dot{x}_{1}=x_{2}$

$\dot{x}_{2}=-\frac{K}{M} x_{1}-\frac{1}{M} F_{c}\left((1+\gamma) x_{2}-w\right)$

$\varepsilon \dot{w}=-w+v_{0}+\gamma x_{2}-\varepsilon \frac{K \gamma}{M} x_{1}$.

The slow variable is $x=\left(x_{1}, x_{2}\right)$, the fast variable is $w$, and the switching function $h$ is $h(x, w)=w-(1+\gamma) x_{2}$.

The slow manifold $\mathcal{M}_{0}(\varepsilon=0$ in the fast subsystem of (C.3)) is the graph of the function $w(x)=v_{0}+\gamma x_{2}$. The first-order approximation of $\mathcal{M}_{ \pm}$is the graph of $w_{ \pm}(x)=v_{0}+\gamma x_{2}+\varepsilon F_{c}\left(x_{2}-v_{0}\right)$. Thus, at the switching surface we have that $w_{ \pm}(x)=v_{0}+\gamma x \mp \varepsilon f_{0} /$ $M$, which means that one effect of the imperfect control (the nonzero $\varepsilon$ ) is a slow-down of the belt velocity of order $\varepsilon$ in $\mathscr{H}_{+}$. The other, less obvious, effect is that the non-zero $\varepsilon$ can limit the minimal length of sliding (sticking) segments that we can observe for the stable stick-slip oscillations. Fig. C.2 shows the approximate return map to the half-line $\mathcal{L}=\left\{(x, w): w=w_{+}(x), x_{2}=0, x_{1}>\right.$ 0 ) close to the grazing-sliding point GS shown in Fig. C.1(c) (again, we have neglected the exponentially small difference between $w$ and $w_{+}(x)$ ). The family of sliding periodic orbits (the stick-slip oscillations) undergo a saddle-node bifurcation and become unstable before the sliding segment shrinks to zero length. This is a consequence of the change of the slope in the graph of the return map in Fig. C.2, a change which is of order 1 uniformly for $\varepsilon \rightarrow 0$. Apparently, the grazing-sliding bifurcation in the dry-friction oscillator falls into case 2 of Lemma 2. Namely, we observe a grazing-sliding bifurcation and the periodic orbit persists, but the stability of the periodic orbit may change completely. The mechanism behind this change is identical to the case $\theta>0$ of the minimal example in Lemma 3. We note that the change of the right-side slopes of the return map in Fig. C. 2 depends on $\gamma$ such that the saddle-node bifurcation occurs only for sufficiently large $\gamma$ (that is, if the belt is not much heavier than the block).

\section{References}

[1] L.N. Virgin, C.J. Begely, Grazing bifurcations and basins of attraction in an impact-friction oscillator, Physica D 130 (1999) 43-57.

[2] S. Banerjee, G. Verghese, Nonlinear Phenomena in Power Electronics, IEEE Press, New York, 2001

[3] F. Dercole, A. Gragnani, S. Rinaldi, Bifurcation analysis of piecewise smooth ecological models, Theoretical Population Biology 72 (2) (2007) 197-213.

[4] M. di Bernardo, C. Budd, A.R. Champneys, P. Kowalczyk, Piecewise-smooth Dynamical Systems - Theory and Applications, in: Applied Mathematical Sciences, vol. 163, Springer-Verlag, 2007.

[5] N. Fenichel, Geometric singular perturbation theory for ordinary differential equations, Journal of Differential Equations 31 (1979) 53-98.

[6] L.M. Fridman, Singularly perturbed analysis of chattering in relay contro systems, IEEE Transactions on Automatic Control 47 (12) (2002) 2079-2084.

[7] L.M. Fridman, Slow periodic motions with internal sliding modes in variable structure systems, Int. J. Control 75 (7) (2002) 524-537.

[8] D.A.W. Barton, B. Krauskopf, R.E. Wilson, Explicit periodic solutions in a model of a relay controller with delay and forcing, Nonlinearity 18 (6) (2005) 2637-2656. 
[9] L. Fridman, E. Fridman, E. Shustin, Steady modes and sliding modes in relay control systems with delay, in: J.P. Barbot, W. Perruquetti (Eds.), Sliding Mode Control in Engineering, Marcel Dekker, New York, 2002, pp. 263-293.

[10] E. Shustin, E. Fridman, L. Fridman, Oscillations in a second-order discontinuous system with delay, Disc. Contin. Dyn. Syst. 9 (2) (2003) 339-358.

[11] R. Szalai, Nonlinear dynamics of high-speed milling, Ph.D. Thesis, Budapest University of Technology and Economics, 2006.

[12] L.M. Fridman, Periodic motions in VSS and singular perturbations, in: X. Yu,
J.-X. Xu (Eds.), Advances in Variable Structure Systems, World Scientific, Singapore, 2000, pp. 365-374.

[13] P.S. Dutta, S. Banerjee, Period increment cascades in a discontinuous map with square-root singularity, in: Proceedings of the 2nd IFAC Conference on analysis and control of chaotic systems, London, UK, 2009.

[14] R. Horváth, Experimental investigation of excited and self-excited vibration, Master's Thesis, University of Technology and Economics, Budapest, 2000, http://www.auburn.edu/ horvaro/index2.html.. 